\title{
The cell-like approximation theorem in dimension 5
}

\author{
by \\ Robert J. Daverman (Knoxville, TN) and \\ Denise M. Halverson (Provo, UT)
}

\begin{abstract}
The cell-like approximation theorem of R. D. Edwards characterizes the $n$-manifolds precisely as the resolvable ENR homology $n$-manifolds with the disjoint disks property for $5 \leq n<\infty$. Since no proof for the $n=5$ case has ever been published, we provide the missing details about the proof of the cell-like approximation theorem in dimension 5 .
\end{abstract}

1. Introduction. The cell-like approximation theorem of R. D. Edwards states that a cell-like mapping $F: M \rightarrow X$ from an $n$-manifold $M$, $n \geq 5$, onto a metric space $X$ can be approximated by homeomorphisms if and only if $X$ is finite-dimensional and has the disjoint disks property. Its fundamentally important corollary is the following characterization of topological manifolds: a (metric) space $X$ is an $n$-manifold, $n \geq 5$, if and only if $X$ is a resolvable ENR homology $n$-manifold having the disjoint disks property.

Edwards outlined the proof in [16], and a fairly complete argument appeared in [11]; both focused on the cases $n>5$. This paper presents an argument concerning the special case $n=5$, a proof for which has never been published, and details of which are not widely known. Since many other applications of the result have been treated elsewhere, we do not discuss those matters here but, instead, concentrate on the proof itself.

The disjoint disks property is untenable in dimensions less than 5, so other properties are needed for a topological characterization of low-dimensional manifolds. Daverman and Repovš [12] have shown that a metric space $X$ is a 3 -manifold if and only if $X$ is a resolvable ENR homology 3-manifold having something called the spherical simplicial approximation property.

2000 Mathematics Subject Classification: Primary 57N15; Secondary 57P05, 57N75.

Key words and phrases: cell-like approximation theorem, disjoint disks, manifold recognition. 
Eliminating the resolvability hypothesis while operating under the assumption that the classical Poincaré conjecture is true, Daverman and Thickstun [13] proved that $X$ is a 3-manifold if and only if it is an ENR homology 3 -manifold having a certain relative simplicial approximation property for 2-complexes. To date no known general position property distinguishes the genuine 4-manifolds among resolvable ENR homology 4-manifolds.

The hypotheses of the cell-like approximation theorem and the characterization of topological manifolds are more essential than recognized when these results were first developed. Dranishnikov produced examples showing that cell-like images of $n$-manifolds, $n \geq 7$, are not necessarily finitedimensional [14], and Dydak and Walsh improved on his constructions for $n \geq 5$ [15]. Bryant, Ferry, Mio and Weinberger have developed examples [4] showing that ENR homology $n$-manifolds, $n \geq 7$, need not be resolvable.

To achieve its main purpose, this paper contributes to decomposition theory by providing conditions under which a cell-like decomposition of a 5manifold can be shrunk out while keeping certain special 2-complexes fixed. Equivalently, it provides conditions under which one cell-like map of a 5manifold to itself can be replaced with another such cell-like map that is the identity on the 2-complex. This is the subject of Section 12. The argument retraces most of the steps required to establish Edwards's cell-like approximation theorem.

The authors are deeply indebted to R. D. Edwards and J. J. Walsh for helpful discussions in times past. The first author first learned a strategy of proof of the 5-dimensional cell-like approximation theorem from Walsh. The argument in the present paper is similar to Walsh's and, it turns out, to Edwards's as well, only with some reduction in the requisite background.

2. Preliminaries. A decomposition $G$ of a space $S$ is upper semicontinuous (usc) if its associated decomposition map $\pi: S \rightarrow S / G$ is proper. Likewise, a proper map $F: S \rightarrow X$ induces an upper semicontinuous decomposition $G=\left\{F^{-1}(x) \mid x \in X\right\}$. The set of all points in $S$ contained in the nondegenerate elements of $G$ is called the nondegeneracy set and is denoted by either $N_{G}$ or $N_{F}$, depending on whether $G$ or $F$ is specified. The union of all elements of $G$ whose diameter is less than $\varepsilon$ will be denoted by $G_{<\varepsilon}$. The union of all elements of $G$ that are single points is the complement of $N_{G}$ and will be denoted as $N_{G}^{c}$. If $U$ is an open set in $S$, then we define

$$
G_{\text {sat }}[U]=\bigcup\{g \in G \mid g \subset U\} .
$$

Upper semicontinuity of $G$ is equivalent to the requirement that, for each open subset $U$ of $S, G_{\text {sat }}[U]$ be open. A set $A \subset S$ is said to be $G$-saturated if $G_{\text {sat }}[A]=A$. 
A set $C$ embedded in an ANR is said to be cell-like if it contracts in every neighborhood of itself. A decomposition for which every decomposition element is cell-like is called a cell-like decomposition. A map $F: M \rightarrow X$ is a cell-like map if the induced decomposition $G=\left\{F^{-1}(x) \mid x \in X\right\}$ is cell-like. A space $X$ is said to be resolvable if there is a cell-like map $F: M \rightarrow X$ where $M$ is a manifold. A space $X$ is an ENR homology $n$-manifold if it can be embedded in some $\mathbb{R}^{m}$ as a neighborhood retract in $\mathbb{R}^{m}$ and it has the local homology of an $n$-manifold.

A metric space $(X, \varrho)$ has the disjoint disks property (DDP) if for any two maps of a 2-disk into $X, \mu_{1}, \mu_{2}: D^{2} \rightarrow X$, and $\varepsilon>0$ there are maps $\mu_{1}^{\prime}, \mu_{2}^{\prime}: D^{2} \rightarrow X$ such that $\varrho\left(\mu_{i}, \mu_{i}^{\prime}\right)<\varepsilon$ and $\mu_{1}^{\prime}\left(D^{2}\right) \cap \mu_{2}^{\prime}\left(D^{2}\right)=\emptyset$.

A set $A \subset S$ is said to be locally $k$-co-connected ( $k$-LCC) in $S$ if for any $x \in A \cap \mathrm{Cl}(S-A)$ and neighborhood $U$ of $x$, there is a neighborhood $V$ of $x$ so that every map of $\partial B^{k+1}$ into $V-A$ can be extended to a map of $B^{k+1}$ into $U-A$. If $A$ is $j$-LCC in $S$ for all $0 \leq j \leq k$, then $A$ is said to be $\mathrm{LCC}^{k}$ in $S$.

The restriction of a map $F: S \rightarrow X$ to $A \subset S$ will be denoted by $F \mid\lceil A\rfloor$. For $A \subset S$, a map $F: S \rightarrow X$ is said to be $1-1$ on $A$ if $F \mid\lceil A\rfloor$ is $1-1$. For $Z \subset X$, a map $F: S \rightarrow X$ is said to be $1-1$ over $Z$ if $F$ is $1-1$ on $F^{-1}(Z)$.

The natural projection maps for a product space $S \times T$ will be denoted as $\Pi_{S}: S \times T \rightarrow S ;(s, t) \rightarrow s$ and $\Pi_{T}: S \times T \rightarrow T ;(s, t) \rightarrow t$.

Given a homotopy $H: Y \times I \rightarrow S$, for each $t \in I$ we define the map $H_{t}: Y \rightarrow S$ such that $H_{t}(x)=H(x, t)$. The level preserving map $\widetilde{H}$ : $Y \times I \rightarrow S \times I$ defined by $\widetilde{H}(x, t)=(H(x, t), t)$ is the lift of $H$. Note that for each level preserving map $\Lambda: Y \times I \rightarrow S \times I$, the map $H=\Pi_{S} \Lambda$ satisfies $\widetilde{H}=\Lambda$. If $H: Y \times I \rightarrow S$ so that $H_{t}$ is a homeomorphism for each $t$, then $H$ is said to be an isotopy. If $H: Y \times I \rightarrow S$ is a homotopy such that $H_{t}$ fails to be a homeomorphism only at $t=1$ then $H$ is said to be a pseudo-isotopy. The map $H_{1}$ is said to be the end of the pseudo-isotopy. If $H: Y \times I \rightarrow S$ is an isotopy (or pseudo-isotopy) and there exists an isotopy (or pseudo-isotopy) $\Lambda: S \times I \rightarrow S$ such that $\Lambda_{0}=$ id and $H=\Lambda \circ\left(H_{0} \times \mathrm{id}\right)$, then $\Lambda$ is said to be a cover of $H$ and $H$ is said to be an ambient isotopy (or an ambient pseudo-isotopy). If $H: Y \times I \rightarrow S$ is an isotopy (or pseudo-isotopy) and there exists an isotopy (or pseudo-isotopy) $\Lambda: S \times I \rightarrow S$ such that $\Lambda_{t_{0}}=$ id and $H=\Lambda \circ\left(H_{t_{0}} \times \mathrm{id}\right)$, then $\Lambda$ is said to be a cover of $H$ respecting $t_{0}$.

For convenience, the image of a path $\gamma: I \rightarrow S$ will be denoted as $\bar{\gamma}$. Given the homotopy $H: Y \times I \rightarrow S$ and $x \in Y$, the path of $x$ by $H$ is the map $\gamma=H \mid\lceil\{x\} \times I\rfloor$. Note that the path of $x$ by $\widetilde{H}$ is $\widetilde{\gamma}$, the lift of the path of $x$ by $H$. The set of points $\bar{\gamma}$ is called the track of $x$ by $H$.

Given a map $\varepsilon: Y \rightarrow(0, \infty)$, then $H: Y \times I \rightarrow S$ is said to be an $\varepsilon(x)$-homotopy if $\operatorname{diam}(H(\{x\} \times I))<\varepsilon(x)$ for all $x \in Y$. If in addition, 
$H$ fixes $Z \subset Y$, i.e., $H_{0}\left|\lceil Z\rfloor=H_{t}\right|\lceil Z\rfloor$ for all $t \in[0,1]$, then $H$ is said to be an $(\varepsilon(x), Z)$-homotopy. If $\pi: S \rightarrow X$ and $\pi H$ is an $\varepsilon(x)$-homotopy, then we say that $H$ is an $\varepsilon(x)[\pi]$-homotopy. If $A, B \subset S$, then an $(\varepsilon, B)$-push of $A$ in $S$ is an $\varepsilon$-isotopy of $S$ into itself that is fixed on $B$ and outside the $\varepsilon$-neighborhood of $A$.

If $M$ is a p.l. $n$-manifold and $A \subset M$, then $A$ has embedding dimension at most $k$, denoted as $\operatorname{dem}(A) \leq k$, if for each $(n-k-1)$-dimensional tamely embedded polyhedron $P$ in $M$ and each open cover $\mathcal{V}$ of $M$, there is a homeomorphism $h: M \rightarrow M$ so that $h(P) \cap A=\emptyset$ and $h$ is $\mathcal{V}$-close to the identity. We say that $A$ has embedding dimension $k$ if $\operatorname{dem}(A) \leq k$ but $\operatorname{not} \operatorname{dem}(A) \leq k-1$.

3. Reduction to the piecewise linear case. For later convenience we will address the cell-like approximation theorem for cell-like maps $F$ : $M \rightarrow X$ defined on compact, p.l. manifolds. In what immediately follows we explain why treatment of that special case disposes of the general case.

Consider a proper, cell-like map $F: M \rightarrow X$ from an $n$-manifold $M$, $n \geq 5$, onto a finite-dimensional space $X$ with the DDP. Given any point $x \in X$, we can produce a compact neighborhood $W_{x} \subset X$ of $x$ such that the inclusion $F^{-1}\left(W_{x}\right) \rightarrow M$ is null-homotopic, by cell-likeness of $F^{-1}(x)$. Cover $X$ by a countable subcollection $\left\{W_{i}\right\}$ of these compact neighborhoods and let $G\left(W_{i}\right)$ be the upper semicontinuous decomposition of $M$ given by the singletons of $M-W_{i}$ and the cell-like sets $F^{-1}(y), y \in W_{i}$. We claim that each $G\left(W_{i}\right)$ is shrinkable. Assuming this claim is true, we will find, using Proposition 23.4 of [11], that $F: M \rightarrow X$ can be approximated, arbitrarily closely, by another proper, cell-like map $F^{\prime}: M \rightarrow X$ which is 1-1 over $\bigcup_{i} W_{i}=X$; the resulting $F^{\prime}$ will be a homeomorphism approximating $F$, as desired.

To establish the claim, we transfer the problem to the p.l. setting. By [17] some neighborhood of $F^{-1}\left(W_{i}\right)$ admits a p.l. triangulation; taking, first, a simplicial neighborhood of $F^{-1}\left(W_{i}\right)$ in some p.l. triangulation and, next, a regular neighborhood of that, we obtain a compact, p.l. $n$-manifold-withboundary $N_{i}$ with $F^{-1}\left(W_{i}\right) \subset \operatorname{int}\left(N_{i}\right) \subset N_{i} \subset M$. The double of $N_{i}$ is a closed, p.l. $n$-manifold $M_{i}$. Specify an embedding $\theta: N_{i} \rightarrow M_{i}$, and cell-like decomposition $G_{i}$ of $M_{i}$ into the singletons of $M_{i}-\theta F^{-1}\left(W_{i}\right)$ and the celllike sets $\theta F^{-1}(y), y \in W_{i}$, with associated decomposition map $\pi_{i}: M_{i} \rightarrow$ $M_{i} / G_{i}$. Since $X$ is finite-dimensional and has the DDP, the same is true of $M_{i} / G_{i}$ (see Exercise 24.11 of [11]). In this p.l. setting, the argument provided here shows that each such map $\pi_{i}: M_{i} \rightarrow M_{i} / G_{i}$ can be approximated by homeomorphisms. In the language of decomposition theory, $G_{i}$ is shrinkable. Furthermore, by Theorem 13.1 of [11], $G_{i}$ is strongly shrinkable; accordingly, there exist homeomorphisms $h_{i}: M_{i} \rightarrow M_{i}$ shrinking the elements of $G_{i}$ to 
small size while fixing all points of $M_{i}-\operatorname{int}\left(\theta\left(N_{i}\right)\right)$. Now $\theta$ can be used to transport such shrinking homeomorphisms of $M_{i}$ to homeomorphisms of $M$ shrinking the elements of $G\left(W_{i}\right)$ to small size while fixing all points of $M-\operatorname{int}\left(N_{i}\right)$. This establishes the shrinkability of $G\left(W_{i}\right)$ and the claim.

4. The strategy of the cell-like approximation theorem. The cell-like approximation theorem states that a cell-like map $F: M \rightarrow X$ defined on an $n$-manifold $M$, where $n \geq 5$, can be approximated by homeomorphisms if and only if $X$ is finite-dimensional and has the disjoint disks property. The strategy of the proof of the cell-like approximation theorem is as follows:

(1) Apply the disjoint disks property to modify the decomposition map so that the embedding dimension of the nondegeneracy set of the modified map is $\leq n-3$.

(2) Filter $X$ into $\sigma$-compact subsets $X=Q^{n}, Q^{n-1}, \ldots, Q^{0}, Q^{-1}=\emptyset$ such that, for $i=0, \ldots n$,

(a) $\operatorname{dim}\left(Q^{i}\right) \leq i$,

(b) $Q^{i} \supseteq Q^{i-1}$,

(c) $\operatorname{dim}\left(Q^{i}-Q^{i-1}\right) \leq 0$.

(3) Set $F_{0}=\pi$; in the sequence of $i=0,1, \ldots, n$, the decomposition induced over $Q^{i}$ is "shrunk out" with control thereby determining a map $F_{i+1}$ approximating $F_{i}$ that is $1-1$ over $Q^{i}$. The result after the $n$th step is a homeomorphism $F=F_{n+1}: M \rightarrow X$ that approximates $\pi$.

The modification of the decomposition map in Step (1) is crucial to the shrinking in Step (3). The shrinking in Step (3) is possible since a 0-dimensional decomposition whose nondegeneracy set has embedding dimension at most $n-3$ can be $\varepsilon$-amalgamated, for any $\varepsilon>0$, to form a decomposition whose elements form a null sequence and have embedding dimension at most $n-3$. The resulting strong cellularity of the amalgamated decomposition elements allows for controlled shrinking of the amalgamated decomposition. The shrinkability of the original decomposition follows from the shrinkability of the $\varepsilon$-amalgamated decompositions. Therefore, the modification of the decomposition map in Step (1) to obtain a decomposition map that has nondegeneracy set having embedding dimension at most $n-3$ is essential to this shrinking argument. The details of the proof are found in [11].

5. The obstacle in the $n=5$ case. The majority of the argument for the proof of the cell-like approximation theorem, found in [11], holds for $n \geq 5$. However, the argument for step (1) is only valid for $n \geq 6$. The result is also claimed for $n=5$, but the proof is not provided. 
What sets the $n=5$ case apart is the fact that homotopic $\mathrm{LCC}^{1}$ embeddings of a 2-complex in a 5-manifold need not be isotopic. (Consider a homotopy in $S^{5}$ from a pair of unlinked 2-spheres to a pair of linked 2 -spheres.) In the case that $n \geq 6$, the following strategy is applied in order to modify the decomposition map so that the nondegeneracy set has embedding dimension at most $n-3$ :

Let $\pi: M \rightarrow M / G$ denote the decomposition map. Suppose that $\left\{\alpha_{i}\right\}$ is a countable dense collection of $\mathrm{LCC}^{1}$ embeddings of finite 2-complexes into the decomposition space $M / G$. Using Proposition 23.5B of [11], the decomposition map is modified to be 1-1 over the images of these embeddings. Assuming that $\pi$ is now the modified decomposition map, let $\alpha_{i}^{*}=\pi^{-1} \alpha_{i}$. Then $\left\{\alpha_{i}^{*}\right\}$ is a set of $\mathrm{LCC}^{1}$ embeddings of 2-complexes in $M$. Therefore, for a given finite $\mathrm{LCC}^{1}$ embedded 2-complex $K \subset M$, there is a controlled homotopy between the inclusion of $K$ and a map in $\left\{\alpha_{i}^{*}\right\}$ (the control is measured in $M / G$ ). The homotopy is piecewise linear (p.l.) adjusted to remove singularities sufficiently so that a controlled ambient isotopy may be obtained. The p.l. adjustment requires that the self-intersection set of the image of the homotopy is of dimension at most 0 . Hence $n \geq 2(\operatorname{dim}(K)+1)$, i.e., $n \geq 6$. The controlled ambient isotopy is used to modify the decomposition map to be 1-1 over the image of $K$. By applying a series of moves guided by controlled ambient isotopies, the decomposition map is modified so that the nondegeneracy set misses an infinite 2-skeleton of $M$, and hence has embedding dimension $\leq n-3$.

Without the assumption that $n \geq 6$, the necessary isotopies may not exist and therefore this particular strategy fails for $n=5$.

6. Overview of the proof in the case $n=5$. In this paper we will demonstrate how to approximate a cell-like map $F: M \rightarrow X$, where $M$ is a 5-manifold, by another cell-like map for which the nondegeneracy set has embedding dimension at most 2 . The proof of the cell-like approximation theorem in dimension 5 will then be complete. (See [11] for details.) In particular, we will prove the following theorem:

Theorem 6.1 (Main Theorem). Suppose $F: M \rightarrow X$ is a cell-like map defined on a 5-manifold $M$ where $\operatorname{dim} X=5$ and $X$ has DDP. Then $F$ can be approximated by a cell-like map $\Psi: M \rightarrow X$ such that the embedding dimension of $N_{\Psi}$ is at most 2.

The procedure for proving the Main Theorem is outlined as follows:

STEP 1. Improve the map $F: M \rightarrow X$ so that $F$ is $1-1$ over a "very large" countable dense collection of $\mathrm{LCC}^{1}$ embedded 2-complexes in $X$. The lifts of the maps in the collection will serve as targets for moves in the next 
step. Now let $F: M \rightarrow X$ denote the improved map and $G$ denote the induced decomposition.

STEP 2. Establish a procedure to move a 2-complex $K$ in $M$ "near" a target. In particular, we show that $K$ may be almost entirely pushed onto a target by an isotopy, the deficit being realized on a finite set of disks in $K$ of arbitrarily small size and $K$ being mapped into $G_{<\varepsilon}$ for some prescribed $\varepsilon$.

STEP 3. Determine an ambient pseudo-isotopy of $M$, the limit of a composition of controlled pushes, the covers of isotopies described in Step 2, such that the end of the ambient pseudo-isotopy $h$ satisfies

(a) $h$ is $1-1$ on $K$ and takes $K$ off $N_{G}$,

(b) $G_{h}=\left\{h^{-1}(x)\right\}$, the decomposition induced by $h$, has nondegeneracy set $N_{h}$ such that $\operatorname{dem}\left(N_{h}\right) \leq 2$.

STEP 4. Apply shrinking fixing a 2-complex to obtain a self-homeomorphism $\Phi: M \rightarrow M$ such that

(a) $\Phi|\lceil K\rfloor=h|\lceil K\rfloor$,

(b) $\Phi=h$ outside $h^{-1}(V)$ where $V$ is a small neighborhood of $h(K)$,

(c) $\varrho(\Phi, h)<\varepsilon$.

STEP 5. Given the 2-skeleta of a sequence $\left\{K_{i}\right\}$ of triangulations whose mesh tends to zero, apply Steps 3 and 4 to determine homeomorphisms $\Phi_{i}: M \rightarrow M$, removing $K_{i}$ from the appropriate nondegeneracy set, and proper cell-like maps $F_{i}=F_{i-1} \Phi_{i}: M \rightarrow X\left(F_{0}=F\right)$ such that the limit map $\Psi$ of $\left\{F_{i}\right\}$ is $1-1$ over the image of the 2-skeleta. Then $\Psi$ will be an approximation of $F$ such that $N_{\Psi}$ has embedding dimension at most 2, our desired result.

7. Improving the decomposition map. In this section we will show how to modify a cell-like map $F: M \rightarrow X$, where $M$ is a 5 -manifold, so that $F$ is 1-1 over a "very large" collection of $\mathrm{LCC}^{1}$ embedded 2-complexes in $X$. These complexes lift to $\mathrm{LCC}^{1}$ embedded complexes in $M$ which act as guides for the controlled moves presented in the next step.

Definition 7.1. A map $F: M \rightarrow X$ is said to be $L C C^{1}$ refined provided that $F$ is 1-1 over a countable collection of $\mathrm{LCC}^{1}$ embedded $k$-complexes in $X, k \leq 2$, denoted by $\mathcal{A}$, satisfying the following:

(1) The Density Property: For any finite $k$-complex $K, k \leq 2, \mathcal{A}$ contains a countable dense subset of the collection of all maps of $K$ into $X$.

(2) The Restriction Property: If $\lambda: K \rightarrow X$ is a map in $\mathcal{A}$ and $L<$ $\operatorname{sd}^{n}(K)$ for some $n \geq 0$, where $\operatorname{sd}^{n}(K)$ is the $n$th barycentric subdivision of $K$, then $\lambda \mid\lceil L\rfloor$ is also a map in $\mathcal{A}$. 
(3) The Extension Property: If $\nu: L \rightarrow X$ is a map in $\mathcal{A}$, and $K$ is a finite $k$-complex, $k \leq 2$, such that $L<K$, then any map $\lambda: K \rightarrow X$ such that $\lambda \mid\lceil L\rfloor=\nu$ can be approximated by a map $\mu: K \rightarrow X$ in $\mathcal{A}$ such that $\mu|\lceil L\rfloor=\lambda|\lceil L\rfloor=\nu$.

In particular, we say that $F$ is $L C C^{1}$ refined over $\mathcal{A}$.

In [11] it is shown that if $X$ is a locally compact separable ANR with DDP and $K$ is a finite 2-complex, then each map $\lambda$ of a finite 2-complex $K$ into $X$ can be approximated by an embedding $\mu: K \rightarrow X$ such that $\mu(K)$ is $\mathrm{LCC}^{1}$ in $X$ [11, Proposition 24.1]. For our purposes here we need a relative version of this result.

Proposition 7.2. Suppose that $X$ is a locally compact separable ANR that has DDP and $\lambda: K \rightarrow X$ is a map defined on a $k$-complex $K, k \leq 2$, and $L$ is a subcomplex of $K$ such that $\lambda \mid\lceil L\rfloor$ is an $L C C^{1}$ embedding. Then $\lambda$ can be approximated by an $L C C^{1}$ embedding $\mu$ so that $\mu|\lceil L\rfloor=\lambda|\lceil L\rfloor$.

Proof. Let $\left\{\left(D_{k}, D_{k}^{\prime}\right)\right\}$ be a countable collection of cell pairs in $K-L$ of dimension at most 2 which separate the points of $K-L$ (that is, for any two points $x, y \in K-L$, there is an index $k$ such that $\left.x \in D_{k}, y \in D_{k}^{\prime}\right)$. Let $\left\{\varsigma_{i}\right\}$ be a countable dense collection of pairwise disjoint $\mathrm{LCC}^{1}$ embeddings of arcs and disks into $X$ missing $\lambda(L)$.

Let $\mathcal{K}=\{\mu: K \rightarrow X|\mu|\lceil L\rfloor=\lambda \mid\lceil L\rfloor\}$. Define $\mathcal{O}_{k}$ to be the set of maps $\mu \in \mathcal{K}$ such that:

(1) $\mu\left(D_{k}\right) \cap \mu\left(D_{k}^{\prime}\right)=\emptyset$,

(2) $\left(\mu\left(D_{k}\right) \cup \mu\left(D_{k}^{\prime}\right)\right) \cap \mu(L)=\emptyset$,

(3) $\mu(K) \cap \bigcup_{i=1}^{k} \operatorname{im}\left(\varsigma_{i}\right)=\emptyset$.

Clearly, $\mathcal{O}_{k}$ is open in $\mathcal{K}$. The fact that $\mathcal{O}_{k}$ is dense in $\mathcal{K}$ follows from the fact that $\lambda(L)$ and $\bigcup_{i=1}^{k} \operatorname{im}\left(\varsigma_{i}\right)$ are $\mathrm{LCC}^{1}$ embedded and that $X$ is an ANR. Let $\mathcal{O}=\bigcap_{k=1}^{\infty} \mathcal{O}_{k}$. For every $\mu \in \mathcal{O}$ :

(1) $\mu|\lceil L\rfloor=\lambda|\lceil L\rfloor$,

(2) $\mu$ is an embedding,

(3) $\mu(K)$ misses $\bigcup_{i=1}^{\infty} \operatorname{im}\left(\varsigma_{i}\right)$ and is therefore an $\mathrm{LCC}^{1}$ embedding. The conclusion of the theorem now follows since $\mathcal{O}$ is dense in $\mathcal{K}$.

Theorem 7.3. Suppose that $F: M \rightarrow X$ is a cell-like map where $X$ is a locally compact separable ANR that has DDP. Then $F$ can be approximated by a cell-like $L C C^{1}$ refined map.

Proof. Note that the set of all finite $k$-complexes, $k \leq 2$, is countable. Therefore, there is a countable dense collection of $\mathrm{LCC}^{1}$ embeddings of finite $k$-complexes in $X$. The set of all restrictions of these maps to the finite subcomplexes of the $n$th barycentric subdivisions, $n=0,1,2, \ldots$, of the 
domain is also countable. Denote the countable collection of embeddings together with their restrictions as $\mathcal{A}_{0}$. We define $\mathcal{A}_{i}$ inductively as follows: Consider a given map $\alpha: K \rightarrow X$ in $\mathcal{A}_{i-1}$. Note that the set of finite complexes $L$ such that $K<L$ is countable. For each $L$, use Proposition 7.2 to determine a countable dense collection, denoted $\mathcal{E}_{i}[\alpha](L)$, of $\mathrm{LCC}^{1}$ embeddings that are extensions of $\alpha$. Let $\mathcal{E}_{i}[\alpha]=\bigcup_{L>K} \mathcal{E}_{i}[\alpha](L)$ and $\mathcal{E}_{i}=$ $\bigcup_{\alpha \in \mathcal{A}_{i-1}} \mathcal{E}_{i}[\alpha]$. Let $\mathcal{A}_{i}$ be the collection of maps in $\mathcal{E}_{i}$ together with their restriction maps to subcomplexes of the $n$th barycentric subdivisions, $n=$ $0,1,2, \ldots$, of the domain. Let

$$
\mathcal{A}=\bigcup_{i=1}^{\infty} \mathcal{A}_{i} .
$$

Then $\mathcal{A}$ is a countable dense collection of $\mathrm{LCC}^{1}$ embeddings in $X$ that satisfies the density, restriction and extension properties.

We claim that $F: M \rightarrow X$ can be approximated arbitrarily closely by a cell-like map $F^{\prime}: M \rightarrow X$ that is 1-1 over $\mathcal{A}$. Briefly describing how this goes, one starts by enumerating the images of the various embeddings from $\mathcal{A}$ as $A_{1}, A_{2}, \ldots$ Then one examines the usc decomposition $G_{1}$ of $M$ induced by $F$ over $A_{1}$; specifically, $G_{1}$ consists of sets of the form $F^{-1}(z), z \in A_{1}$, together with the singletons of $M-F^{-1}\left(A_{1}\right)$. The possible nonmanifold set of the associated decompositions space, $M / G_{1}$, is contained in the 2dimensional polyhedron homeomorphic to $A_{1}$ and equal to the image of $F^{-1}\left(A_{1}\right) \approx A_{1}$, which one can readily see is 1 -LCC embedded in $M / G_{1}$, using lifting properties of cell-like, proper mappings. According to [23], $G_{1}$ is shrinkable. Thus, there exists a cell-like map $\mu_{1}: M \rightarrow M$ such that $\left\{\mu_{1}^{-1}(Z) \mid z \in M\right\}=G_{1}$ and $F_{1}=F \mu_{1}^{-1}$ is a close approximation to $F$. Note that $F_{1}: M \rightarrow X$ is a cell-like map which is 1-1 over $A_{1}$.

Repeat the process, using $F_{1}$ in place of $F$, to obtain a new cell-like map $F_{2}$ approximating $F_{1}$ which is $1-1$ over $A_{1} \cup A_{2}$. Since $F_{1}$ is already 1-1 over $A_{1}$, one can do the shrinking that determines $F_{2}$ while keeping points of $F_{1}^{-1}\left(A_{1}\right)$ fixed, so that $F_{1}$ and $F_{2}$ agree on $F_{1}^{-1}\left(A_{1}\right)=F_{2}^{-1}\left(A_{1}\right)$. Continue in this fashion, obtaining successive cell-like maps $F_{k}: M \rightarrow X$ which are 1-1 over $\bigcup_{i=1}^{k} A_{i}$, Just as in the proof of [11, Theorem 23.2], one can impose controls on the successive approximations $F_{k}$ to ensure that $\lim _{k \rightarrow \infty} F_{k}$ is a cell-like map $F^{\prime}: M \rightarrow X$ that is $1-1$ over $\bigcup_{i} A_{i}$. Therefore $F^{\prime}$ is an approximation of $F$ that is $\mathrm{LCC}^{1}$ refined over $\mathcal{A}$.

8. Isotopies and covers. In this section we note some results about isotopies and covers that will be needed in later sections. It follows from the first result, due to Bryant and Seebeck [5], that it will be sufficient to establish our technology in the p.l. setting. The second theorem is due to Hudson [19] and the third is a controlled version of the same result. The fourth 
is primarily attributed to Connelly [10] and Miller [20] with contributions made by Cobb [8], Akin [1], and Bryant and Seebeck [5] (see [3]).

Theorem 8.1 (Bryant-Seebeck). Suppose $f: K \rightarrow M$ is an $L C C^{1}$ embedding of a $k$-dimensional polyhedron $K$ into a p.l. $n$-manifold $M$, and $n-k \geq 3, n \geq 5$. Then for every $\varepsilon>0$, there is an $\varepsilon$-push $H$ of $f(K)$ such that $H_{1} f$ is p.l.

TheOrem 8.2 (Hudson). Suppose that $K$ is a compact $k$-dimensional polyhedron, $M$ is a p.l. $n$-manifold, and $n-k \geq 3$. Then any p.l. isotopy of $K$ in $M$ is ambient.

Given such a p.l. isotopy, after taking a general position approximation as in [2] (see also [24]), one can obtain a controlled analog of the Hudson ambient isotopy result.

THeOREM 8.3. Suppose that $K$ is a compact $k$-dimensional polyhedron, $M$ is a p.l. n-manifold where $n-k \geq 3, \kappa>0, h: K \times I \rightarrow M$ is a p.l. isotopy such that $\operatorname{diam}(h(x \times I))<\kappa$ for all $x \in K$, and $W$ is a neighborhood of $h(K \times I)$. Then there exists an ambient isotopy $H: M \times I \rightarrow M$ supported in $W$ such that the track of each point under $H$ has diameter less than $n \kappa$ and $H h_{0}=h_{1}$.

Theorem 8.4 (Miller, Connelly). Suppose that $K$ is a $k$-dimensional compact polyhedron, $M$ is a p.l. n-manifold, and $f: K \rightarrow M$ is a proper topological embedding, $n-k \geq 3$. Then for every $\varepsilon>0$ there is a $\delta>0$ such that if $\lambda_{i}: K \rightarrow M$ are p.l. embeddings, $i=0,1$, within $\delta$ of $f$, then there is an $\varepsilon$-push $H$ of $f(K)$ in $M$ such that $H \lambda_{0}=\lambda_{1}$.

Next, we establish a basic fact about covers:

Lemma 8.5. If $\Lambda$ is a cover of $H$ respecting $t_{1}$ then $\Lambda^{\prime}=\Lambda\left(\Lambda_{t_{2}}^{-1} \times \mathrm{id}\right)$ is a cover of $H$ respecting $t_{2}$.

Proof. Suppose that $\Lambda$ is a cover of $H$ respecting $t_{1}$. Then $\Lambda_{t_{1}}=\mathrm{id}$ and $\Lambda \circ\left(H_{t_{1}} \times \mathrm{id}\right)=H$. Let $\Lambda^{\prime}=\Lambda\left(\Lambda_{t_{2}}^{-1} \times \mathrm{id}\right)$. Note that $\Lambda_{t_{2}}^{\prime}=\Lambda_{t_{2}} \Lambda_{t_{2}}^{-1}=\mathrm{id}$. Furthermore, $H_{t_{2}}=\Lambda_{t_{2}} H_{t_{1}}$. Hence

$\Lambda^{\prime} \circ\left(H_{t_{2}} \times \mathrm{id}\right)=\Lambda\left(\Lambda_{t_{2}}^{-1} \times \mathrm{id}\right) \circ\left(H_{t_{2}} \times \mathrm{id}\right)=\Lambda \circ\left(\Lambda_{t_{2}}^{-1} H_{t_{2}} \times \mathrm{id}\right)=\Lambda \circ\left(H_{t_{1}} \times \mathrm{id}\right)=H$.

Therefore $\Lambda_{t_{1}}^{\prime}=\mathrm{id}$ and $\Lambda^{\prime} \circ\left(H_{t_{2}} \times \mathrm{id}\right)=H$ so $\Lambda^{\prime}$ is a cover of $H$ respecting $t_{2}$.

Lemma 8.5 motivates the following definition.

Definition 8.6. If $\Lambda$ is a cover of an isotopy $H$, then the $t_{0}$-shift of $\Lambda$ is the isotopy $\Lambda^{\prime}=\Lambda\left(\Lambda_{t_{0}}^{-1} \times \mathrm{id}\right)$. 
9. Singularity structures and structure maps. The failure of homotopic $\mathrm{LCC}^{1}$ embeddings of a 2-complex $K$ in a 5 -manifold $M$ to be isotopic is illustrated in Figure 1. The picture is of the lift $\widetilde{H}: K \times I \rightarrow M \times I$ of a homotopy $H: K \times I \rightarrow M$ in general position. Note that the embeddings of the 2-complex $K$ in $M \times\{0\}$ and $M \times\{1\}$ appear 1-dimensional, but the nature of the problem is correctly represented. In this case, the paths of two distinct 2 -simplexes in $K$ cross in a single point $p$. Due to dimension constraints, this intersection cannot always be removed. In the $n \geq 6$ case of the cell-like approximation theorem, where a decomposition $G$ of $M$ is specified, the extra dimension allows for the removal of such a singularity so that $K$ can be pushed to the end of $H$, which is determined to miss $N_{G}$. We will demonstrate that in the $n=5$ case, the goal of pushing $K$ off $N_{G}$ may be accomplished through a sequence of pushes that end "near" target 2-complexes embedded in $M$ away from the nondegeneracy set.

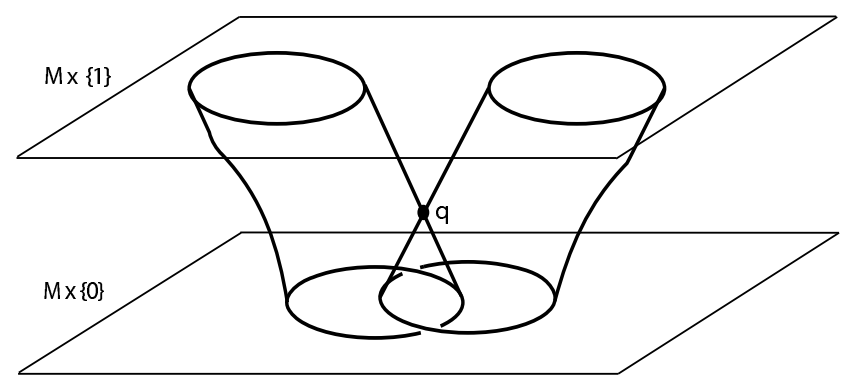

Fig. 1. The obstruction to adjusting a homotopy to an isotopy. The 2-complex $K$ is represented by two disjoint copies of $S^{1}$.

In this section, we identify structures associated with singularities of homotopies that will serve as guides for modifications in the next section that will produce nearby isotopies. In particular, we will do the following:

(1) Define singularity structures for p.l. level preserving maps $\widetilde{H}: K \times$ $I \rightarrow M \times I$ whose singularities consist of isolated double points.

(2) Use the singularity structures to define an isotopy $J: K^{*} \times I \rightarrow M$, where $K^{*}$ is the complex formed by identifying points $x_{i}$ and $y_{i}$ in $K$ that correspond to the double points $\left(x_{i}, t_{i}\right)$ and $\left(y_{i}, t_{i}\right)$ of $\widetilde{H}$ : $K \times I \rightarrow M \times I$.

(3) Show that the isotopy $J$ can be further approximated by a p.l. isotopy, with sufficient controls to achieve the goals of the next sections.

It is the p.l. condition that requires extra special care and is the ultimate goal of this section. The p.l. condition will be needed later in the application of Theorem 8.3. 
Definition 9.1. Suppose that $\widetilde{H}: K \times I \rightarrow M \times I$ is a p.l. level preserving map so that $q=\widetilde{H}\left(x, t_{0}\right)=\widetilde{H}\left(y, t_{0}\right)$ and the tracks of $x$ and $y$ by $\widetilde{H}$ in $M \times I$ meet only at $q$. Then an artifact map for $q$ is a p.l. level preserving map $\psi: I \times I \rightarrow M \times I$ such that

(1) $\psi(0, t)=\widetilde{H}(x, t)$,

(2) $\psi(1, t)=\widetilde{H}(y, t)$,

(3) $\psi\left(I \times\left\{t_{0}\right\}\right)=q$,

(4) $\psi \mid\left\lceil I \times\left(I-\left\{t_{0}\right\}\right)\right\rfloor$ is an embedding,

(5) $\psi \mid\left\lceil(I-\partial I) \times\left(I-\left\{t_{0}\right\}\right)\right\rfloor \operatorname{misses} \operatorname{im}(\widetilde{H})$.

If $\beta$ denotes the path $\psi \mid\lceil I \times\{1\}\rfloor$ that connects $\widetilde{H}(x, 1)$ to $\widetilde{H}(y, 1)$ in $M \times$ $\{1\}$, then we say that the artifact map ends in $\beta$ or ends at $\bar{\beta}$ (see Figure 2 ).

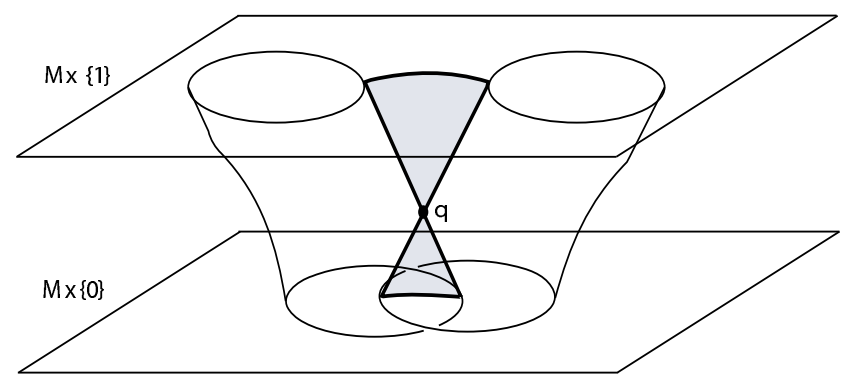

Fig. 2. An artifact map. The shaded region is $\operatorname{im}(\psi)$.

Definition 9.2. Suppose that $H: K \times I \rightarrow M$ is a homotopy so that the lift $\widetilde{H}: K \times I \rightarrow M \times I$ is a level preserving p.l. map in general position such that the following hold:

(a) The only singularities of $\widetilde{H}$ are double points occurring at $\left\{q_{1}, \ldots, q_{m}\right\}$ $\subset M \times I$ such that for $q_{i}=\widetilde{H}\left(x_{i}, t_{i}\right)=\widetilde{H}\left(y_{i}, t_{i}\right),\left\{x_{1}, \ldots, x_{m}\right.$, $\left.y_{1}, \ldots, y_{m}\right\}$ is a set of distinct points in $K$.

(b) For each $q_{i}$, there is an artifact map $\psi_{i}: I \times I \rightarrow M \times I$.

(c) The images of the maps $\psi_{i}$ are mutually disjoint.

Then $H$ is a well-behaved homotopy and the collection of artifact maps is said to be a singularity structure for $H$.

The following result demonstrates the existence of singularity structures for well-behaved homotopies.

Proposition 9.3. Suppose that $\widetilde{H}: K \times I \rightarrow M \times I$ is a level preserving p.l. map in general position so that $q=\widetilde{H}\left(x, t_{0}\right)=\widetilde{H}\left(y, t_{0}\right)$ and the tracks of $x$ and $y$ by $\widetilde{H}$ in $M \times I$ meet only at $q$. Let $\gamma_{1}$ and $\gamma_{2}$ denote the paths 
of $x$ and $y$ by $\widetilde{H}$, respectively. Suppose that $V$ is an open set in $M$ and $\beta: I \rightarrow V \times\{1\}$ is a p.l. embedding connecting $\widetilde{H}(x, 1)$ to $\widetilde{H}(y, 1)$ such that $Q=\bar{\beta} \cup \bar{\gamma}_{1} \cup \bar{\gamma}_{2}$ contracts in $V \times I$. Then there is an artifact map $\psi: I \times I \rightarrow V \times I$ for $q$ ending at $\bar{\beta}$. Moreover, for any prescribed p.l. 3-complex in $M \times I$, we may assume that $\operatorname{im}(\psi)$ misses the 3 -complex away from $Q$.

Proof. Since $Q$ contracts in $V \times I$, it is straightforward to determine a map $\omega: I \times I \rightarrow V \times I$ satisfying

(1) $\omega(0, t)=\widetilde{H}(x, t)$,

(2) $\omega(1, t)=\widetilde{H}(y, t)$,

(3) $\omega\left(I \times\left\{t_{0}\right\}\right)=q$,

(4) $\omega(s, 1)=\beta(s)$.

Define $\phi=\left(\Pi_{V} \circ \omega\right) \times \Pi_{I}$, and note that $\phi$ is level preserving. Approximate $\phi$ by a p.l. level preserving map fixed on the 1-complex $(\partial I \times I) \cup\left(I \times\left\{t_{0}, 1\right\}\right)$ and supported in $V$. General position may then be applied to remove any unnecessary singularities and any intersections with a prescribed 3-complex away from $Q$ while preserving the levels. The resulting map $\psi$ is the desired artifact map.

Definition 9.4. Suppose that $H: K \times I \rightarrow M$ is a well-behaved homotopy with a singularity structure specified as in Definition 9.2 and $\phi: M \times I \rightarrow M \times I$ is a map such that

(1) $\phi$ is level preserving,

(2) $\phi=$ id outside $N\left(\bigcup_{i=1}^{m} \operatorname{im}\left(\psi_{i}\right) ; \varepsilon\right)$,

(3) $\phi=$ id on each $N\left(\operatorname{im}\left(\psi_{i}\right) ; \varepsilon\right) \cap\left(M \times t_{i}\right)$,

(4) $\phi=$ id on $\widetilde{H}\left(\left\{y_{1}, \ldots, y_{m}\right\} \times I\right)$,

(5) $\left\{\psi_{i}(I \times t) \mid t \in I-\left\{t_{i}\right\}\right.$ and $\left.i=1, \ldots, m\right\}$ are the only nondegenerate elements of the decomposition $G_{\phi}$ induced by $\phi$.

Then $\phi$ is said to be an $(H, \varepsilon)$-buttressing map.

Proposition 9.5. Suppose that $H: K \times I \rightarrow M$ is a well-behaved homotopy with singularity structure specified as in Definition 9.2 (or 9.1). Then for any $\varepsilon>0$ there exists an $(H, \varepsilon)$-buttressing map. Moreover, if $L<K$ such that $\left\{x_{1}, \ldots, x_{m}\right\} \subset K-L$ and $\left\{y_{1}, \ldots, y_{m}\right\} \subset L$, we may require that the $(H, \varepsilon)$-buttressing map is the identity on $H(L \times I)$.

Proof. Let $H$ and $L$ be given as in the hypothesis (if not otherwise specified, let $\left.L=\left\{y_{1}, \ldots, y_{m}\right\}\right)$. Without loss of generality, suppose that $\varepsilon$ is sufficiently small so that the $\varepsilon$-neighborhoods of $\operatorname{im}\left(\psi_{i}\right)$ are disjoint. Let $G^{*}$ be the decomposition of $M \times I$ with nondegeneracy set $N_{G^{*}}=\left\{\psi_{i}(I \times t) \mid\right.$ $t \in I-\left\{t_{i}\right\}$ and $\left.i=1, \ldots, m\right\}$ (see Figure 3 ). It follows from the p.l. structure 


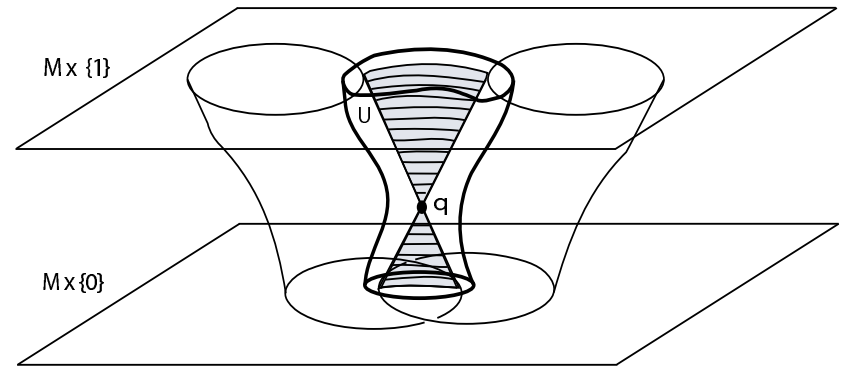

Fig. 3. A level preserving decomposition induced by an artifact map.

of $H$ and $\psi_{i}$ that $G^{*}$ is strongly shrinkable preserving levels and fixing $\widetilde{H}(L \times I)$. (Section 12 provides details reinforcing this statement.) Hence the decomposition map $\pi^{*}: M \times I \rightarrow(M \times I) / G^{*}$ is a (level preserving) near-homeomorphism. Thus there is a level preserving homeomorphism $h$ : $(M \times I) / G^{*} \rightarrow M \times I$ so that for $\phi=h \pi^{*}$,

(1) $\phi=$ id outside $N\left(\bigcup_{i=1}^{m} \operatorname{im}\left(\psi_{i}\right) ; \varepsilon\right)$,

(2) $\phi=\operatorname{id}$ on $N\left(\operatorname{im}\left(\psi_{i}\right) ; \varepsilon\right) \cap\left(M \times t_{i}\right)$,

(3) $\phi=$ id on $\widetilde{H}(L \times I)$.

Then $\phi$ is level preserving and $\left\{\psi_{i}(I \times t) \mid t \in I-\left\{t_{i}\right\}\right.$ and $\left.i=1, \ldots, m\right\}$ are the only nondegenerate elements of the decomposition $G_{\phi}$ induced by $\phi$. Thus $\phi$ is an $(H, \varepsilon)$-buttressing map.

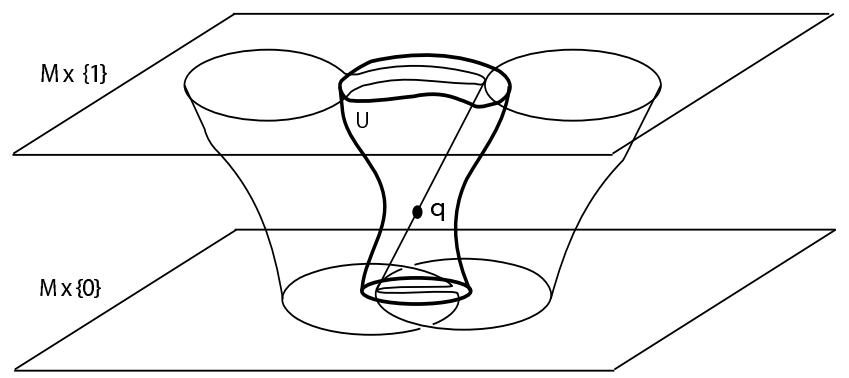

Fig. 4. The lift of an isotopy $J: K^{*} \times I \rightarrow M$ that is $U$-near $H$ respecting the singularity at $q$.

Definition 9.6. Suppose that $H: K \times I \rightarrow M$ is a well-behaved homotopy with singularity structure specified as in Definition 9.2. Let $\theta$ denote the quotient map defined on $K$ that identifies $x_{i}$ and $y_{i}$. Let $K^{*}$ be the complex that is the image of $\theta$. Let $U$ be an open set containing $\bigcup_{i=1}^{m} \operatorname{im}\left(\psi_{i}\right)$. Then $J: K^{*} \times I \rightarrow M$ is an isotopy $U$-near $H$ respecting the singularities $\left\{q_{1}, \ldots, q_{m}\right\}$ if for $i=1, \ldots, m$ there are neighborhoods $U_{i} \subset U$ of $\operatorname{im}\left(\psi_{i}\right)$ 
such that

$$
J \circ(\theta \times \mathrm{id})=H \quad \text { on } \quad H^{-1}\left(\left[(M \times I)-\bigcup_{i=1}^{m} U_{i}\right] \cup \bigcup_{i=1}^{m}\left[\left(M \times\left\{t_{i}\right\}\right) \cap U_{i}\right]\right)
$$

(see Figure 4).

Proposition 9.7. Suppose that $H: K \times I \rightarrow M$ is a well-behaved homotopy with singularity structure as specified in Definition 9.2. Then for any $\varepsilon>0$ and $L<K$ such that $\left\{x_{1}, \ldots, x_{m}\right\} \subset K-L$ and $\left\{y_{1}, \ldots, y_{m}\right\} \subset L$, there is an isotopy $J: K^{*} \times I \rightarrow M$ that is $N\left(\bigcup_{i=1}^{m} \operatorname{im}\left(\psi_{i}\right) ; \varepsilon\right)$-near $H$ and $J \circ(\theta \times \mathrm{id})=H$ on $L \times I$.

Proof. By Proposition 9.5 there is an $(H, \varepsilon)$-buttressing map $\phi$ that is the identity on $H(L \times I)$. Let $J=\phi H \circ\left(\theta^{-1} \times\right.$ id $)$. Note that $J \circ(\theta \times$ id $)=H$ on $L \times I$. Hence $J$ is the desired isotopy.

REMARK 9.8. One should note here that the isotopy $J$ found in the proof of Theorem 9.7 is not p.l., primarily due to the failure of $\phi=h \pi^{*}$ to be a p.l. map. To see this, recall that for $m \geq 2$, a map from $\mathbb{R}^{m}$ to itself whose only nontrivial point preimage is a segment cannot be p.l. Thus, in general, even the level maps of $J$ fail to be p.l. We must do extra work to overcome this difficulty. because the p.l. condition aids in ensuring the existence of a cover. Therefore, it will be desirable to approximate $J$ by a p.l. map that is again $N\left(\bigcup_{i=1}^{m} \operatorname{im}\left(\psi_{i}\right) ; \varepsilon\right)$-near $H$ without moving points of $L \times I$. The next propositions will establish the parameters in which the adjustment is made.

Proposition 9.9. Let $K$ be a compact $k$-dimensional polyhedron, $L$ a subpolyhedron of $K$, and $M$ an n-manifold such that $n-k \leq 3$. Suppose that $J: K \times I \rightarrow M$ is an isotopy so that $\widetilde{J} \mid\lceil L \times I\rfloor$ is p.l. and $\varepsilon>0$. Then there is a p.l. isotopy $J^{\prime}: K \times I \rightarrow M$ such that

(1) $J=J^{\prime}$ on $L \times I$,

(2) $\varrho\left(J, J^{\prime}\right)<\varepsilon$,

(3) $J=J^{\prime}$ on any finite set of levels for which $J$ is p.l.

Proof. We will first show the theorem is true in the case that $J$ is constant on $L$. Using a relative version of Theorem 8.4, for each $t \in I$, there is a $\delta_{t}$ such that if $f_{1}, f_{2}: K \rightarrow M$ are p.l. embeddings $\delta_{t}$ close to $J_{t}$ and agreeing with $J_{t}$ on $L$, then $f_{1}$ and $f_{2}$ are p.l. $(\varepsilon, L)$-isotopic. Let $\eta$ be a Lebesgue number for $\left\{N\left(t, \delta_{t}\right)\right\}$. Choose $0=t_{0}<t_{1}<\cdots<t_{n-1}<t_{n}=1$ so that $\varrho\left(J_{t_{i+1}}, J_{t_{i}}\right)<\eta / 3$. The set of levels $\left\{t_{0}, t_{1}, \ldots, t_{n}\right\}$ should include the finite set of levels for which $J$ is specified to be p.l. from the hypotheses. If $J_{t_{i}}$ is p.l., define $\lambda_{t_{i}}=J_{t_{i}}$. Otherwise, let $\lambda_{t_{i}}$ be an $\eta / 3$-approximation of $J_{t_{i}}$ by a p.l. embedding that agrees with $J_{t_{i}}$ on $L$. Thus $\varrho\left(\lambda_{t_{i+1}}, \lambda_{t_{i}}\right)<\eta / 3$. Then $\lambda_{t_{i+1}}$ and $\lambda_{t_{i}}$ are p.l. $(\varepsilon, L)$-isotopic. The homotopy product $J^{\prime}$ of these isotopies is a p.l. isotopy satisfying the conclusion. 
To prove the general case, apply Theorem 8.2 to determine a p.l. cover $\Lambda$ of the p.l. isotopy $J \mid\lceil L \times I\rfloor$. Note that $\widetilde{\Lambda}$ and $\widetilde{\Lambda}^{-1}$ are p.l. level preserving homeomorphisms. Let $J^{*}=\Pi_{M} \widetilde{\Lambda}^{-1} \widetilde{J}$. It follows from the fact $J \mid\lceil L\rfloor=$ $\Lambda \circ\left(J_{0} \mid\lceil L\rfloor \times\right.$ id $)$ that

$$
J^{*}\left|\lceil L\rfloor=\Pi_{M} \widetilde{\Lambda}^{-1} \tilde{\Lambda} \circ\left(J_{0} \times \mathrm{id}\right)=J_{0}\right|\lceil L\rfloor .
$$

Thus $J^{*}$ is an isotopy fixed on $L$. Choose $\xi>0$ so that if $\operatorname{diam} A<\xi$, then $\widetilde{\Lambda}(A)<\varepsilon$. It follows from the special case proved above that there is a p.l. isotopy $J^{* *}$ fixed on $L$ such that $\varrho\left(J^{* *}, J^{*}\right)<\xi$ and $J^{* *}=J^{*}$ on the specified levels. Then the isotopy $J^{\prime}$ defined by $\widetilde{J}^{\prime}=\widetilde{\Lambda} \widetilde{J}^{* *}$ is the desired isotopy.

Theorem 9.10. Suppose that $H: K \times I \rightarrow M$ is a well-behaved homotopy with singularity structure specified as in Definition 9.2. Then for any $\varepsilon>0$ and $L<K$ such that $\left\{x_{1}, \ldots, x_{m}\right\} \subset K-L,\left\{y_{1}, \ldots, y_{m}\right\} \subset L$, and $H \mid\lceil L \times I\rfloor$ is a p.l. map, there is a p.l. isotopy $J: K^{*} \times I \rightarrow M$ that is $N\left(\bigcup_{i=1}^{m} \operatorname{im}\left(\psi_{i}\right) ; \varepsilon\right)$-near $H$ so that $J \circ(\theta \times \mathrm{id})=H$ on $L \times I$. Moreover, if $\phi: M \times I \rightarrow M \times I$ is an $(H, \varepsilon)$-buttressing map fixed on $\widetilde{H}(L \times I)$ so that $\phi H$ is p.l. on $K \times\left\{t_{1}, \ldots, t_{m}\right\}$, we may require that $\widetilde{J} \circ(\theta \times \mathrm{id})=\phi \widetilde{H}$ on $K \times\left\{t_{1}, \ldots, t_{m}\right\}$.

Proof. Let $L^{\prime}<K$ so that $L<L^{\prime},\left\{x_{1}, \ldots, x_{m}\right\} \subset K-L^{\prime}$ and $\widetilde{H}\left(\overline{K-L^{\prime}} \times I\right)$ $\subset N\left(\bigcup_{i=1}^{m} \operatorname{im}\left(\psi_{i}\right) ; \varepsilon\right)$. By Proposition 9.7 there is an isotopy $J^{\prime}: K^{*} \times I \rightarrow M$ that is $N\left(\bigcup_{i=1}^{m} \operatorname{im}\left(\psi_{i}\right) ; \varepsilon\right)$-near $H$ so that $J^{\prime} \circ(\theta \times$ id $)=H$ on $L^{\prime} \times I$. Note that $\widetilde{J}^{\prime} \circ(\theta \times \mathrm{id})\left(\overline{K-L^{\prime}} \times I\right) \subset N\left(\bigcup_{i=1}^{m} \operatorname{im}\left(\psi_{i}\right) ; \varepsilon\right)$. Choose $\eta>0$ so that $N\left(\widetilde{J}^{\prime} \circ(\theta \times \operatorname{id})\left(\overline{K-L^{\prime}} \times I\right) ; \eta\right) \subset N\left(\bigcup_{i=1}^{m} \operatorname{im}\left(\psi_{i}\right) ; \varepsilon\right)$. By Proposition 9.9, there is a p.l. isotopy $J: K \times I \rightarrow M$ such that

(1) $J=J^{\prime}$ on $L^{\prime} \times I$, and

(2) $\varrho\left(J, J^{\prime}\right)<\eta$.

Note that $J$ is $N\left(\bigcup_{i=1}^{m} \operatorname{im}\left(\psi_{i}\right) ; \varepsilon\right)$-near $H$ and $J \circ(\theta \times$ id $)=H$ on $L^{\prime} \times I$. Therefore, $J$ is the desired isotopy.

To satisfy the "moreover" condition, let $\phi$ be as in the formulation of the theorem and $J^{\prime}=\phi \widetilde{H} \circ\left(\theta^{-1} \times \mathrm{id}\right)$. By applying Proposition 9.9, we may obtain a p.l. isotopy $J: K^{*} \times I \rightarrow M$ approximating $J^{\prime}$ so that

(1) $J=J^{\prime}=H \circ(\theta \times$ id $)$ on $L \times I$,

(2) $J$ is sufficiently close to $J^{\prime}$ so that $J$ is also $N\left(\bigcup_{i=1}^{m} \operatorname{im}\left(\psi_{i}\right) ; \varepsilon\right)$ near $H$,

(3) $J \circ(\theta \times$ id $)=J^{\prime} \circ(\theta \times$ id $)=\phi H$ on $K \times\left\{t_{1}, \ldots, t_{m}\right\}$.

It follows that $J$ is the desired p.l. isotopy. 
10. Moving a 2-complex "near" a target. Given a well-behaved homotopy with singularity occurring at $q=\widetilde{H}\left(x, t_{0}\right)=\widetilde{H}\left(y, t_{0}\right)$, our strategy will be to identify a particularly nice thin product neighborhood within an $\varepsilon$-neighborhood of the image of an artifact map for $q$. The product neighborhood will allow us to determine a modification of $\widetilde{H}$, by a replacement procedure within the $\varepsilon$-neighborhood, that removes the singularity at $q$. In the setting of the next section where a decomposition is specified, it will be shown that for any $\varepsilon>0$ it can be arranged that the corresponding modified $H$ ends in $G_{<\varepsilon}$ on a neighborhood of $x$ and ends in $N_{G}^{c}$ elsewhere.

Our first theorem provides a key step to the replacement procedure detailed in the final theorem of this section.

Theorem 10.1. Suppose that $H: K \times I \rightarrow M$ is a homotopy such that the lift $\widetilde{H}: K \times I \rightarrow M \times I$ is a level preserving p.l. map in general position with one singularity, a double point, at $q=\widetilde{H}\left(x, t_{0}\right)=\widetilde{H}\left(y, t_{0}\right)\left(t_{0} \neq 0,1\right)$, and $\psi$ is an artifact map for $q$. Then for any $\varepsilon>0$ and disjoint p.l. disks $D, D^{\prime} \subset K$ such that $x \in \operatorname{int}(D), y \in \operatorname{int}\left(D^{\prime}\right)$, and $\widetilde{H}\left(\left(D \cup D^{\prime}\right) \times I\right) \subset$ $N(\operatorname{im}(\psi) ; \varepsilon)$, there are p.l. isotopies $e: B^{5} \times I \rightarrow M$ and $\Gamma: M \times I \rightarrow M$ so that

(1) $\left(\partial D \cup D^{\prime}\right) \times I \subset \widetilde{H}^{-1}(\operatorname{im}(\widetilde{e})) \subset\left(D \cup D^{\prime}\right) \times I$,

(2) $\operatorname{im}(\psi) \subset \operatorname{im}(\widetilde{e}) \subset N(\operatorname{im}(\psi) ; \varepsilon)$,

(3) $\Gamma$ is a cover of $H \mid\lceil\overline{K-D} \times I\rfloor$ respecting $t_{0}$,

(4) $\Gamma$ is a cover of e respecting $t_{0}$.

Furthermore, if $J: K^{*} \times I \rightarrow M$ is an isotopy $N(\operatorname{im}(\psi) ; \varepsilon)$-near $H$ so that $J \circ(\theta \times \mathrm{id})=H$ on $\overline{K-D} \times I, \Lambda$ is a cover of $J$ respecting $t_{0}, \phi: M \times I \rightarrow$ $M \times I$ is an $(H, \varepsilon)$-buttressing map, and $\Omega$ is an open set containing $\operatorname{im}(\psi)$, then we may require that $\widetilde{\Gamma} \widetilde{\Lambda}^{-1}=\mathrm{id}$ on $[(M \times I)-N(\operatorname{im}(\psi) ; \varepsilon)] \cup\left[M \times\left\{t_{0}\right\}\right]$ and $\widetilde{\Gamma} \widetilde{\Lambda}^{-1} \phi=$ id on $(M \times I)-\Omega$.

Proof. Let $D$ and $D^{\prime}$ be disjoint p.l. disks in $K$ as above. Let $J: K^{*} \times I \rightarrow$ $M$ be a p.l. isotopy $N(\operatorname{im}(\psi) ; \varepsilon)$-near $H$ so that

$$
J \circ(\theta \times \text { id })=H \quad \text { on } \overline{K-D} \times I
$$

and let $\Lambda$ be a cover of $J$ respecting $t_{0}$. Note that $J$ and $\Lambda$ are given or may be constructed by applying Theorem 9.10. Moreover,

$$
J=\Lambda \circ\left(J_{t_{0}} \times \mathrm{id}\right) .
$$

By Theorem 9.5, there is an $(H, \varepsilon)$-buttressing map $\phi$ that is the identity on $H(\overline{K-D} \times I)$.

Let $B$ be a p.l. 5-ball in $M \times\left\{t_{0}\right\}$ so that $q \in \operatorname{int}(B) \times\left\{t_{0}\right\}$ and $\theta^{-1} J_{t_{0}}^{-1}(B)=D \cup D^{\prime}$. Such a $B$ may be found by taking a small regular 
neighborhood of $H\left(\left(D \cup D^{\prime}\right) \times\left\{t_{0}\right\}\right)$ in $M \times\left\{t_{0}\right\}$. Let

$$
T=\phi^{-1} \widetilde{\Lambda}(B \times I) .
$$

Without loss of generality we may assume that $B$ is sufficiently small so that

$$
T \subset N(\operatorname{im}(\psi) ; \varepsilon) .
$$

Choose $\varepsilon^{\prime}>0$ so that $N\left(\operatorname{im}(\psi) ; \varepsilon^{\prime}\right) \subset T$ or $N\left(\operatorname{im}(\psi) ; \varepsilon^{\prime}\right) \cap \Omega \subset T$ if $\Omega$ is given. Let $\phi^{\prime}$ be an $\left(H, \varepsilon^{\prime}\right)$-buttressing map. Let $\widetilde{e}: B^{5} \times I \rightarrow M \times I$ be defined by

$$
\widetilde{e}=\phi^{\prime} \phi^{-1} \widetilde{\Lambda} \circ(j \times \mathrm{id})
$$

where $j: B^{5} \rightarrow B$ is a p.l. homeomorphism. Note that since $\operatorname{im}(\widetilde{e})=T$, then

$$
\operatorname{im}(\psi) \subset \operatorname{im}(\widetilde{e}) \subset N(\operatorname{im}(\psi) ; \varepsilon) .
$$

Since on $\left(\partial D \cup D^{\prime}\right) \times I, J \circ(\theta \times \mathrm{id})=H$ and $\phi=\mathrm{id}$, we have

$$
\begin{aligned}
\widetilde{H}\left(\left(\partial D \cup D^{\prime}\right) \times I\right) & =\phi^{-1} \widetilde{J} \circ(\theta \times \mathrm{id})\left(\left(\partial D \cup D^{\prime}\right) \times I\right) \\
& =\phi^{-1} \widetilde{\Lambda} \circ\left(J_{t_{0}} \times \mathrm{id}\right) \circ(\theta \times \mathrm{id})\left(\left(\partial D \cup D^{\prime}\right) \times I\right) \\
& =\phi^{-1} \widetilde{\Lambda} \circ\left(J_{t_{0}} \theta\left(\partial D \cup D^{\prime}\right) \times I\right) \subset \phi^{-1} \widetilde{\Lambda}(B \times I)=T=\operatorname{im}(\widetilde{e}) .
\end{aligned}
$$

Also,

$$
\widetilde{H}\left(\left(K-D \cup D^{\prime}\right) \times I\right) \cap \operatorname{im}(\widetilde{e})=\phi^{-1} \widetilde{J}\left(\left(K-D \cup D^{\prime}\right) \times I\right) \cap T=\emptyset .
$$

Hence

$$
\widetilde{H}^{-1}(\operatorname{im}(\widetilde{e})) \subset D \cup D^{\prime} \times I .
$$

Therefore, $\widetilde{e}$ is the desired embedding.

Now define $\widetilde{\Gamma}=\phi^{\prime} \phi^{-1} \widetilde{\Lambda}$. It is clear that $\Gamma_{t_{0}}$ is the identity map since $\phi^{\prime} \phi^{-1}$ and $\widetilde{\Lambda}$ are the identity map on the $t_{0}$ level. Note also that

$$
\begin{aligned}
J_{t_{0}} \theta \mid\lceil\overline{K-D}\rfloor & =J \circ(\theta \times \mathrm{id}) \mid\left\lceil\overline{K-D} \times\left\{t_{0}\right\}\right\rfloor \\
& =\Pi_{M} \widetilde{J} \circ(\theta \times \mathrm{id})\left|\left\lceil\overline{K-D} \times\left\{t_{0}\right\}\right\rfloor=\Pi_{M} \phi \widetilde{H}\right|\left\lceil\overline{K-D} \times\left\{t_{0}\right\}\right\rfloor \\
& =\Pi_{M} \circ\left(\phi \mid\left\lceil M \times\left\{t_{0}\right\}\right\rfloor\right) \circ\left(\widetilde{H} \mid\left\lceil\overline{K-D} \times\left\{t_{0}\right\}\right\rfloor\right) \\
& =\Pi_{M} \widetilde{H}\left|\left\lceil\overline{K-D} \times\left\{t_{0}\right\}\right\rfloor=H\right|\left\lceil\overline{K-D} \times\left\{t_{0}\right\}\right\rfloor=H_{t_{0}} \mid\lceil\overline{K-D}\rfloor .
\end{aligned}
$$

Hence

$$
\begin{aligned}
& \widetilde{\Gamma} \circ\left(H_{t_{0}} \mid\lceil\overline{K-D}\rfloor \times \mathrm{id}\right) \\
&=\phi^{\prime} \phi^{-1} \widetilde{\Lambda} \circ\left(H_{t_{0}} \mid\lceil\overline{K-D}\rfloor \times \mathrm{id}\right) \\
&=\phi^{\prime} \phi^{-1} \widetilde{J}\left(J_{t_{0}} \times \mathrm{id}\right)^{-1} \circ\left(H_{t_{0}} \mid\lceil\overline{K-D}\rfloor \times \mathrm{id}\right) \\
&=\phi^{\prime} \phi^{-1} \widetilde{J}\left(J_{t_{0}}^{-1} \times \mathrm{id}\right) \circ\left(H_{t_{0}} \mid\lceil\overline{K-D}\rfloor \times \mathrm{id}\right) \\
&=\phi^{\prime} \phi^{-1} \phi \widetilde{H} \circ\left(\theta^{-1} \times \mathrm{id}\right) \circ\left(J_{t_{0}}^{-1} \times \mathrm{id}\right) \circ\left(H_{t_{0}} \mid\lceil\overline{K-D}\rfloor \times \mathrm{id}\right)
\end{aligned}
$$




$$
\begin{aligned}
& =\phi^{\prime} \widetilde{H} \circ\left(\theta^{-1} J_{t_{0}}^{-1} H_{t_{0}} \mid\lceil\overline{K-D}\rfloor \times \mathrm{id}\right) \\
& =\phi^{\prime} \widetilde{H} \circ\left(\left(\theta^{-1} J_{t_{0}}^{-1} H_{t_{0}} \times \mathrm{id}\right) \mid\lceil\overline{K-D} \times I\rfloor\right) \\
& =\phi^{\prime} \widetilde{H}|\lceil\overline{K-D} \times I\rfloor=\widetilde{H}|\lceil\overline{K-D} \times I\rfloor .
\end{aligned}
$$

Therefore

$$
H \mid\lceil\overline{K-D} \times I\rfloor=\Gamma \circ\left(H_{t_{0}} \mid\lceil\overline{K-D}\rfloor \times \mathrm{id}\right)
$$

and so $\Gamma$ is a cover of $H \mid\lceil\overline{K-D} \times I\rfloor$ respecting $t_{0}$.

Note also that

$$
\begin{aligned}
e_{t_{0}} & =\Pi_{M} \phi^{\prime} \phi^{-1} \widetilde{\Lambda}(j \times \mathrm{id}) \mid\left\lceil B^{5} \times\left\{t_{0}\right\}\right\rfloor \\
& =\Pi_{M} \circ\left(\phi^{\prime} \phi^{-1} \widetilde{\Lambda} \mid\left\lceil M \times\left\{t_{0}\right\}\right\rfloor\right) \circ\left((j \times \mathrm{id}) \mid\left\lceil B^{5} \times\left\{t_{0}\right\}\right\rfloor\right) \\
& =\Pi_{M} \circ\left((j \times \mathrm{id}) \mid\left\lceil B^{5} \times\left\{t_{0}\right\}\right\rfloor\right)=j
\end{aligned}
$$

and hence

$$
\widetilde{\Gamma}\left(e_{t_{0}} \times \mathrm{id}\right)=\widetilde{\Gamma}(j \times \mathrm{id})=\phi^{\prime} \phi^{-1} \widetilde{\Lambda} \circ(j \times \mathrm{id})=\widetilde{e} .
$$

Therefore

$$
e=\Gamma \circ\left(e_{t_{0}} \times \mathrm{id}\right)
$$

and so $\Gamma$ is a cover of $e$ respecting $t_{0}$.

Further note that $\widetilde{\Gamma} \widetilde{\Lambda}^{-1}=\phi^{\prime} \phi^{-1}=$ id on $[(M \times I)-N(\operatorname{im}(\psi) ; \varepsilon)] \cup[M \times$ $\left.\left\{t_{0}\right\}\right]$ and $\widetilde{\Gamma} \widetilde{\Lambda}^{-1} \phi=\phi^{\prime}=\mathrm{id}$ on $(M \times I)-\Omega$. In conclusion, $\Gamma$ is the desired cover of $H \mid\lceil\overline{K-D} \times I\rfloor$ and $e$.

Theorem 10.2. Suppose that $H: K \times I \rightarrow M$ is a well-behaved homotopy such that the lift $\widetilde{H}: K \times I \rightarrow M \times I$ is a level preserving p.l. map in general position with one singularity, a double point, at $q=\widetilde{H}\left(x, t_{0}\right)=\widetilde{H}\left(y, t_{0}\right)$ $\left(t_{0} \neq 0,1\right)$, and $\psi$ is an artifact map for $q$. Then for any $\varepsilon>0$ there exist a p.l. disk $D$ in $K$ and an isotopy $H^{\prime}: K \times I \rightarrow M$ so that

(1) $x \in D$,

(2) $\operatorname{diam}(D)<\varepsilon$,

(3) $\widetilde{H}^{\prime}(D \times I) \subset N(\operatorname{im}(\psi) ; \varepsilon)$,

(4) $H^{\prime}=H$ on $\overline{K-D} \times I$,

(5) $H_{0}^{\prime}=H_{0}$.

Furthermore, if $J: K^{*} \times I \rightarrow M$ is an isotopy $N(\operatorname{im}(\psi) ; \varepsilon)$-near $H$ so that $J \circ(\theta \times \mathrm{id})=H$ on $\overline{K-D} \times I$ and $\Lambda$ is a cover of $J$ respecting $t_{0}, \phi: M \times I \rightarrow$ $M \times I$ is an $(H, \varepsilon)$-buttressing map and $\Omega$ is an open set containing $\operatorname{im}(\psi)$, then we may require that $H^{\prime}$ is covered by an isotopy $\Gamma: M \times I \rightarrow M$ respecting $t_{0}$ so that $\widetilde{\Gamma} \widetilde{\Lambda}^{-1}=\mathrm{id}$ on $[(M \times I)-N(\operatorname{im}(\psi) ; \varepsilon)] \cup\left[M \times\left\{t_{0}\right\}\right]$ and $\widetilde{\Gamma} \widetilde{\Lambda}^{-1} \phi=\mathrm{id}$ on $(M \times I)-\Omega$. 
Proof. Let $\varepsilon>0$. Let $D, D^{\prime}$ be p.l. disks in $K$ such that $x \in \operatorname{int}(D)$, $y \in \operatorname{int}\left(D^{\prime}\right), \operatorname{diam}(D)<\varepsilon$ and $\widetilde{H}\left(\left(D \cup D^{\prime}\right) \times I\right) \subset N(\operatorname{im}(\psi) ; \varepsilon)$. Let $J$ : $K^{*} \times I \rightarrow M$ be an isotopy $N(\operatorname{im}(\psi) ; \varepsilon)$-near $H$ so that $J \circ(\theta \times \mathrm{id})=H$ on $\overline{K-D} \times I$ and let $\Lambda$ be a cover of $J$ respecting $t_{0}$. Note that $J$ and $\Lambda$ are given or may be constructed by applying Theorem 9.10. By Theorem 10.1 there are p.l. isotopies $e: B^{5} \times I \rightarrow M$ and $\Gamma: M \times I \rightarrow M$ so that

(1) $\left(\partial D \cup D^{\prime}\right) \times I \subset \widetilde{H}^{-1}(\operatorname{im}(\widetilde{e})) \subset\left(D \cup D^{\prime}\right) \times I$,

(2) $\operatorname{im}(\psi) \subset \operatorname{im}(\widetilde{e}) \subset N(\operatorname{im}(\psi) ; \varepsilon)$,

(3) $\Gamma$ is a cover of $H \mid\lceil\overline{K-D} \times I\rfloor$ respecting $t_{0}$,

(4) $\Gamma$ is a cover of $e$ respecting $t_{0}$,

(5) $\widetilde{\Gamma} \widetilde{\Lambda}^{-1}=\mathrm{id}$ on $[(M \times I)-N(\operatorname{im}(\psi) ; \varepsilon)] \cup\left[M \times\left\{t_{0}\right\}\right]$ and $\widetilde{\Gamma} \widetilde{\Lambda}^{-1} \phi=\mathrm{id}$ on $(M \times I)-\Omega$.

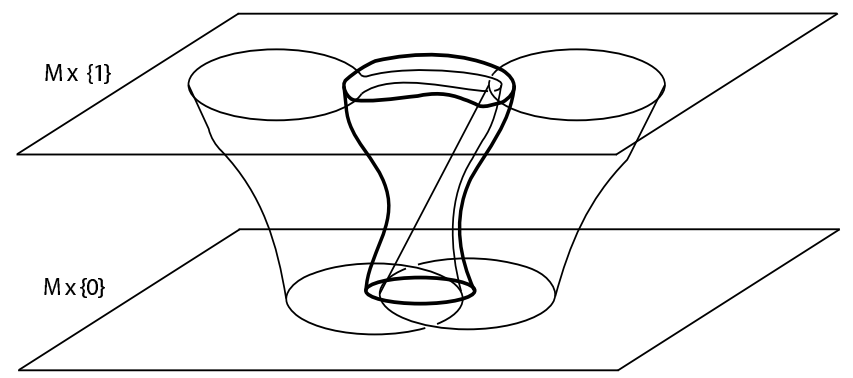

Fig. 5. The result of a replacement procedure

Our strategy will be to remove the intersection point $q$ by replacing the map $\widetilde{H} \mid\lceil D \times I\rfloor$ with an embedding into $N(\operatorname{im}(\psi) ; \varepsilon)$ that does not meet $\widetilde{H} \mid\lceil\overline{K-D} \times I\rfloor($ see Figure 5$)$. Let $Q=\Pi_{B^{5}} \circ(\widetilde{e})^{-1} \widetilde{H}(D \times 0)$. Let $\mu$ : $D \times I \rightarrow Q \times I$ be such that $\mu(x, t)=(s, t)$ where $\widetilde{e}(s, 0)=\widetilde{H}(x, 0)$. Note that $\widetilde{H}|\lceil\partial D \times I\rfloor=\widetilde{e} \mu|\lceil\partial D \times I\rfloor$. Now let $\widetilde{H}^{\prime}=\widetilde{H} \cup \widetilde{e} \mu$. Note that $\widetilde{H}^{\prime}(D \times I) \subset$ $N(\operatorname{im}(\psi) ; \varepsilon), H^{\prime}=H$ on $\overline{K-D} \times I$, and $H_{0}^{\prime}=H_{0}$. Also note that the map $\Gamma: M \times I \rightarrow M$ is a cover of $H^{\prime}$ respecting $t_{0}$ since it is a cover of $H \mid[\overline{K-D} \times I\rfloor$ respecting $t_{0}$ and a cover of $e$ respecting $t_{0}$, or in particular a cover of $e \mu$ respecting $t_{0}$. By construction, $H_{0}^{\prime}=H_{0}$. Then $H^{\prime}$ and $\Gamma$ are the desired isotopies.

Corollary 10.3. Suppose that $H: K \times I \rightarrow M$ is a well-behaved homotopy so that the lift $\widetilde{H}: K \times I \rightarrow M \times I$ is a level preserving p.l. map in general position such that the following hold:

(a) The only singularities of $\widetilde{H}$ are double points occurring at $\left\{q_{1}, \ldots, q_{m}\right\}$ $\subset M \times I$ such that for $q_{i}=\widetilde{H}\left(x_{i}, t_{i}\right)=\widetilde{H}\left(y_{i}, t_{i}\right),\left\{x_{1}, \ldots, x_{m}\right.$, $\left.y_{1}, \ldots, y_{m}\right\}$ is a set of distinct points in $K$.

(b) For each $q_{i}$, there is an artifact map $\psi_{i}: I \times I \rightarrow M \times I$. 
(c) The images of the maps $\psi_{i}$ are mutually disjoint.

(d) There are mutually disjoint open sets $\left\{U_{1}, \ldots, U_{m}\right\}$ so that $U_{i}$ is a neighborhood of $\operatorname{im}\left(\psi_{i}\right)$.

Then for any $\varepsilon>0$ there is a finite set $\left\{D_{1}, \ldots, D_{m}\right\}$ of disjoint p.l. disks in $K$ and an isotopy $H^{\prime}: K \times I \rightarrow M$ so that

(1) $x_{i} \in D_{i}$,

(2) $\operatorname{diam}\left(D_{i}\right)<\varepsilon$

(3) $\widetilde{H}^{\prime}\left(D_{i} \times I\right) \subset N\left(\operatorname{im}\left(\psi_{i}\right) ; \varepsilon\right)$,

(4) $H^{\prime}=H$ on $\overline{K-\bigcup_{i=1}^{m} D_{i}} \times I$,

(5) $H_{0}^{\prime}=H_{0}$.

Furthermore, if $N\left(\operatorname{im}\left(\psi_{i}\right) ; \varepsilon\right) \subset U_{i}$ and $J: K^{*} \times I \rightarrow M$ is an isotopy $N(\mathrm{im}(\psi) ; \varepsilon)$-near $H$ so that $J \circ(\theta \times \mathrm{id})=H$ on $\overline{K-\bigcup_{i=1}^{m} D_{i}} \times I, \Lambda$ is a cover of $J, \phi: M \times I \rightarrow M \times I$ is an $(H, \varepsilon)$-buttressing map, and $\Omega$ is an open set containing $\bigcup_{i=1}^{m} \operatorname{im}(\psi)_{i}$, then we may require that $H^{\prime}$ is covered by an isotopy $\Gamma: M \times I \rightarrow M$ respecting the singularities of $\widetilde{H}$ so that $\widetilde{\Gamma} \widetilde{\Lambda}^{-1}=\mathrm{id}$ on $\left[(M \times I)-N\left(\bigcup \operatorname{im}\left(\psi_{i}\right) ; \varepsilon\right)\right] \cup\left[\bigcup_{i=1}^{m}\left(U_{i} \cap\left(M \times\left\{t_{i}\right\}\right)\right)\right]$ and $\widetilde{\Gamma} \widetilde{\Lambda}^{-1} \phi=\mathrm{id}$ on $(M \times I)-\Omega$.

Proof. Without loss of generality, assume $\varepsilon>0$ is given so that $N\left(\operatorname{im}\left(\psi_{i}\right) ; \varepsilon\right)$ $\subset U_{i}$ for each $i=1, \ldots, m$. Choose p.l. disks $D_{i}, D_{i}^{\prime} \subset K$ so that $\operatorname{diam}\left(D_{i}\right)$ $<\varepsilon$ and $\widetilde{H}\left(\left(D_{i} \cup D_{i}^{\prime}\right) \times I\right) \subset N\left(\operatorname{im}\left(\psi_{i}\right) ; \varepsilon\right)$. Let $J: K^{*} \times I \rightarrow M$ be an isotopy as in the statement, and let $\Lambda$ be a cover of $J: K^{*} \times I \rightarrow M$. Both $J$ and $\Lambda$ are specified or may be constructed by applying Theorem 9.10.

We will proceed inductively on $i=1, \ldots, m$ by performing a replacement procedure to remove each singularity, one at a time. Let $K[i]$ be the complex formed from $K$ by attaching $x_{j}$ to $y_{j}$ for $j>i$. Note that $K[0]=K^{*}$. Let $\theta[i]: K \rightarrow K[i]$ denote the attaching map. Let $\Theta[i]: K[i] \rightarrow K[i]^{*}=K[i-1]$ denote the map that attaches $x_{i}$ to $y_{i}$ in $K[i]$. Define $\phi[i]=\phi$ on $U_{i}$ and $\phi[i]=\mathrm{id}$ otherwise. Note that $\phi=\phi[1] \ldots \phi[m]$.

CASE $i=1$. Define $H[1]: K[1] \times I \rightarrow M$ so that $H[1]=H \circ\left(\theta[1]^{-1} \times\right.$ id $)$ on $D_{1} \times I$ and $H[1]=J \circ(\Theta[1] \times$ id $)$ on $\left(K[1]-D_{1}\right) \times I$. Then $\widetilde{H}[1]$ is a p.l. map with one singularity at $q_{1}=\widetilde{H}\left(x_{1}, t_{1}\right)=\widetilde{H}\left(y_{1}, t_{1}\right)$ and $\psi_{1}$ is an artifact map for $q_{1}$. Let $J[1]=J$ and note that $K[1]^{*}=K[0]=K^{*}$. Hence $J[1]$ is an isotopy of $K[1]^{*}$ that is $N\left(\operatorname{im}\left(\psi_{1}\right) ; \varepsilon\right)$-near $H[1]$ so that $J[1] \circ(\Theta[1] \times \mathrm{id})=H[1]$ on $\left(K[1]-D_{1}\right) \times I$. Let $\Lambda[1]$ be the $t_{1}$-shift of $\Lambda$. Then $\Lambda[1]$ is a cover of $J[1]$ respecting $t_{1}$. Thus $\phi[1]$ is an $(H[1], \varepsilon)$-buttressing map. By Theorem 10.2 there exist an isotopy $H^{\prime}[1]: K[1] \times I \rightarrow M$ and a disk $D_{1}$ in $K$ so that

(1) $x \in D_{1}$,

(2) $\operatorname{diam}\left(D_{1}\right)<\varepsilon$, 
(3) $\widetilde{H}^{\prime}[1]\left(D_{1} \times I\right) \subset N\left(\operatorname{im}\left(\psi_{1}\right) ; \varepsilon\right)$,

(4) $H^{\prime}[1]=H[1]$ on $\left(K-D_{1}\right) \times I$,

(5) $H^{\prime}[1]_{0}=H[1]_{0}$.

Furthermore, we may require that $H^{\prime}[1]$ is covered by an isotopy $\Gamma[1]: M \times I$ $\rightarrow M$ respecting $t_{1}$ so that $\widetilde{\Gamma}[1] \widetilde{\Lambda}[1]^{-1}=$ id on $\left[(M \times I)-N\left(\operatorname{im}\left(\psi_{1}\right) ; \varepsilon\right)\right] \cup$ $\left[U_{1} \cap\left(M \times\left\{t_{1}\right\}\right)\right]$ and $\widetilde{\Gamma}_{1} \widetilde{\Lambda}_{1}^{-1} \phi[1]=\mathrm{id}$ on $(M \times I)-\Omega$.

CASE $i>1$. Define $H[i]: K[i] \times I \rightarrow M$ so that $H[i]=H \circ\left(\theta[i]^{-1} \times\right.$ id $)$ on $D_{i} \times I$ and $H[i]=H^{\prime}[i-1]$ on $\left(K[i]-D_{i}\right) \times I$. Then $\widetilde{H}[i]$ is a p.l. map with one singularity at $q_{i}=\widetilde{H}\left(x_{i}, t_{i}\right)=\widetilde{H}\left(y_{i}, t_{i}\right)$ and $\psi_{i}$ is an artifact map for $q_{i}$. Let $J[i]=H^{\prime}[i-1]$ and note that $K[i]^{*}=K[i-1]$. Hence $J[i]$ is an isotopy of $K[i]^{*}$ that is $N\left(\operatorname{im}\left(\psi_{i}\right) ; \varepsilon\right)$-near $H[i]$ so that $J[i] \circ(\Theta[i] \times \mathrm{id})=H[i]$ on $\left(K[i]-D_{i}\right) \times I$. Let $\Lambda[i]$ be the $t_{i}$-shift of $\Gamma[i-1]$. Then $\Lambda[i]$ is a cover of $J[i]$ respecting $t_{i}$. Thus $\phi[i]$ is an $(H[i], \varepsilon)$-buttressing map. By Theorem 10.2 there exist an isotopy $H^{\prime}[i]: K[i] \times I \rightarrow M$ and a disk $D_{i}$ in $K$ so that

(1) $x \in D_{i}$,

(2) $\operatorname{diam}\left(D_{i}\right)<\varepsilon$

(3) $\widetilde{H}^{\prime}[i]\left(D_{i} \times I\right) \subset N\left(\operatorname{im}\left(\psi_{i}\right) ; \varepsilon\right)$,

(4) $H^{\prime}[i]=H[i]$ on $\left(K-D_{i}\right) \times I$,

(5) $H^{\prime}[i]_{0}=H[i]_{0}$.

Furthermore, we may require that $H^{\prime}[i]$ is covered by an isotopy $\Gamma[i]: M \times I$ $\rightarrow M$ respecting $t_{i}$ so that $\widetilde{\Gamma}[i] \widetilde{\Lambda}[i]^{-1}=$ id on $\left[(M \times I)-N\left(\operatorname{im}\left(\psi_{i}\right) ; \varepsilon\right)\right] \cup$ $\left[U_{i} \cap\left(M \times\left\{t_{i}\right\}\right)\right]$ and $\widetilde{\Gamma}_{i} \widetilde{\Lambda}_{i}^{-1} \phi[i]=$ id on $(M \times I)-\Omega$.

At the $m$ th step, $K[m]=K$. The desired isotopy is $H^{\prime}=H^{\prime}[m]$ and the desired cover $\Gamma$ is the 0 -shift of $\Gamma[m]$.

11. Moving a 2-complex off the nondegeneracy set. In this section, our focus will be on a 5-manifold $M$ and a cell-like usc decomposition $G$ such that $M / G$ is finite-dimensional and has DDP. The map $\pi: M \rightarrow M / G$ will denote the associated decomposition map. In view of Theorem 7.3, we may assume that $\pi$ is $\mathrm{LCC}^{1}$ refined over a collection $\mathcal{A}$ of $\mathrm{LCC}^{1}$ embeddings satisfying the density, restriction and extension properties of the definition of a $\mathrm{LCC}^{1}$ refined map. The collection of lifts of maps in $\mathcal{A}$ will be denoted by $\mathcal{A}^{*}$. In particular

$$
\mathcal{A}^{*}=\left\{\alpha^{*} \mid \alpha^{*}=\pi^{-1} \alpha, \alpha \in \mathcal{A}\right\} .
$$

Note that $\mathcal{A}^{*}$ is a countable collection of $\mathrm{LCC}^{1}$ embeddings of 2 -complexes in $M$.

Given a 2-complex $K$ in $M$ we desire to push $K$ off the nondegeneracy set $N_{G}$ by a pseudo-isotopy that is small as viewed in $M / G$. This will be accomplished by a sequence of moves that takes $K$ successively closer to 
a controlled limit of maps in $\mathcal{A}^{*}$. The following theorem will be key to obtaining the control needed for these pushes.

TheOREM 11.1. Let $\pi: M \rightarrow X$ be a cell-like map of a compact p.l. $n$ manifold $M$ onto a finite-dimensional metric space $X$. Then for every $\varepsilon>0$, there is a $\delta>0$ so that if $f, f^{\prime}: K \rightarrow M$ are $L C C^{1}$ embeddings of a finite $k$-complex $K$, where $k \leq n-3$ and $H: K \times I \rightarrow M$ is a $(\delta, L)[\pi]$-isotopy between $f$ and $f^{\prime}$ supported in an open set $U$, then there is an $\varepsilon[\pi]$-push $\Lambda: M \times I \rightarrow M$ of $f(\overline{K-L})$ supported in $U$ that is a cover of $H$.

This theorem follows from 8.3 and the techniques established by Miller in [20] with special care taken to measure controls in the decomposition space.

The pseudo-isotopy sought for in this section will be comprised of a sequence of moves which are detailed in the following theorem.

TheOREM 11.2. Let $G$ be a cell-like usc decomposition of a 5-manifold $M$ with associated decomposition map $\pi: M \rightarrow M / G$ such that $\pi$ is $L C C^{1}$ refined over $\mathcal{A}$. Suppose $K$ is a finite 2 -complex and $f^{*}: K \rightarrow M$ is an $L C C^{1}$ embedding such that $f^{*}(K) \subset G_{<\eta}$. Furthermore, suppose that $L<K$ and $L^{\prime}$ is a simplicial neighborhood of $L$ in some $n$th barycentric subdivision of $K$ such that $f^{*} \mid\left\lceil L^{\prime}\right\rfloor \in \mathcal{A}^{*}$. Then for any $\varepsilon>0$ and $G$-saturated neighborhood $U^{*}$ of $f^{*}\left(\overline{K-L^{\prime}}\right)$, there exist a finite set $\mathcal{D}$ consisting of disjoint p.l. disks in $K-L^{\prime}$ and an isotopy $\Gamma: M \times I \rightarrow M$ so that

(1) $\Gamma$ is supported in $U^{*}$,

(2) $\Gamma \circ\left(f^{*} \times\right.$ id $)$ is a $\left(4 \eta, L^{\prime}\right)$-isotopy,

(3) $\Gamma$ is also an $\varepsilon[\pi]$-isotopy,

(4) $\Gamma$ pushes $f^{*}(K)$ into $G_{<\varepsilon}$,

(5) for $D \in \mathcal{D}, \operatorname{diam}(D)<\varepsilon$,

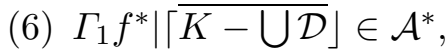

(7) if $D \in \mathcal{D}$, then $\pi \Gamma_{1} f^{*}(D) \cap \pi \Gamma_{1} f^{*}(K-D)=\emptyset$,

(8) for all $D \in \mathcal{D}, \operatorname{diam}\left(\Gamma_{1} f^{*}(D)\right)<4 \eta$.

Moreover, if $Z$ is a compact subset of $M$ that misses $f^{*}(K)$ and $\pi(Z)$ is $L C C^{1}$, we may also require that $\pi \Gamma_{1}(Z) \cap \pi \Gamma_{1} f^{*}(K)=\emptyset$.

Proof. Let $\varepsilon>0$ and $U^{*}$ be a $G$-saturated neighborhood of $\overline{K-L^{\prime}}$ missing $L$. Choose $\delta>0(\delta<\varepsilon / 2)$ to satisfy Theorem 11.1 for $\varepsilon$. Let $f=\pi f^{*}$.

Our first goal is to identify an embedding of $\lambda: K \rightarrow M / G$ in $\mathcal{A}$ sufficiently close to $f^{*}$ to act as a target for the desired isotopy. Define

$$
V^{*}=\pi^{-1}\left(N\left(f\left(\overline{K-L^{\prime}}\right) ; \delta\right)\right) \quad \text { and } \quad W^{*}=G_{\text {sat }}\left[N\left(f^{*}\left(\overline{K-L^{\prime}}\right) ; \eta\right)\right] .
$$

Note that $W^{*}$ is a neighborhood of $f^{*}\left(\overline{K-L^{\prime}}\right)$ since $f^{*}(K) \subset G_{<\eta}$. Let

$$
O^{*}=U^{*} \cap V^{*} \cap W^{*} .
$$


In particular, $O^{*}$ is the intersection of $G$-saturated neighborhoods of $f^{*}\left(\overline{K-L^{\prime}}\right)$ and is therefore a $G$-saturated neighborhood of $f^{*}\left(\overline{K-L^{\prime}}\right)$. Thus $O=\pi\left(O^{*}\right)$ is a neighborhood of $f\left(\overline{K-L^{\prime}}\right)$.

Let $3 \zeta<\delta$ be a Lebesgue number for the cover

$$
\mathcal{F}=\left\{\pi\left(G_{\text {sat }}[N(x ; \eta)]\right) \cap O \mid x \in f^{*}\left(\overline{K-L^{\prime}}\right)\right\}
$$

of $f\left(\overline{K-L^{\prime}}\right)$ in $M / G$. Let $\lambda \in \mathcal{A}$ such that $\lambda$ is $\left(\zeta, L^{\prime}\right)$-homotopic to $f$ and define $\lambda^{*}=\pi^{-1} \lambda$. Then there is an approximate lift to a $\left(\zeta, L^{\prime}\right)[\pi]$-homotopy $H: K \times I \rightarrow O^{*}$ such that $H_{0}=f^{*}$ and $H_{1}=\lambda^{*}$, which we also note is a $\left(2 \eta, L^{\prime}\right)$-homotopy. We may assume that $\widetilde{H}$ is in general position so that the singular set consists of double points occurring at $\left\{q_{1}, \ldots, q_{m}\right\} \subset M \times I$. We may also assume that the double points have distinct $M$-coordinates so that for $\widetilde{H}\left(x_{i}, t_{i}\right)=\widetilde{H}\left(y_{i}, t_{i}\right)=q_{i}$, the elements of $\left\{x_{1}, y_{1}, \ldots, x_{m}, y_{m}\right\}$ are distinct. It follows that the track of $x_{i}$ by $\widetilde{H}$ meets only the track of $y_{i}$ by $\widetilde{H}$ and vice versa, the intersection occurring at $q_{i}$. Without loss of generality, we may also assume that $x_{i}$ and $y_{i}$ are the vertices of some $n$th barycentric subdivision of $K$. Note that since $H$ is supported in an open set away from $L$, neither $x_{i}$ nor $y_{i}$ are in $L$. Since $H$ is constant on $L^{\prime}$, at least one of $x_{i}$ or $y_{i}$ is in $K-L^{\prime}$ for each $i$. By relabeling points if necessary, we impose the condition that $x_{i} \in K-L^{\prime}$.

Our next goal is to construct the guiding structure for our isotopy. We begin by defining artifact maps for each $q_{i}$. Let $\alpha_{i}=H \mid\left\lceil\left\{x_{i}\right\} \times I\right\rfloor$ and $\beta_{i}=H \mid\left\lceil\left\{y_{i}\right\} \times I\right]$. Let

$$
E_{i}^{*}=\pi^{-1}\left(N\left(\pi \Pi_{M}\left(q_{i}\right) ; \zeta\right)\right) \subset O^{*} \cap G_{\text {sat }}\left[N\left(\Pi_{M}\left(q_{i}\right) ; 2 \eta\right)\right] .
$$

Note that $\bar{\alpha}_{i} \cup \bar{\beta}_{i} \subset E_{i}^{*}$. Let $\gamma_{i}: I \rightarrow M$ be a p.l. path connecting $H\left(x_{i}, 1\right)$ to $H\left(y_{i}, 1\right)$, and otherwise missing $H(K \times\{1\})$ so that $\bar{\alpha}_{i} \cup \bar{\beta}_{i} \cup \bar{\gamma}_{i}$ contracts in $E_{i}^{*}$. By the extendability condition on $\mathcal{A}$ we may, and do, require that $\lambda^{*} \cup \bigcup_{i=1}^{m} \gamma_{i} \in \mathcal{A}^{*}$. By Proposition 9.3, there are artifact maps $\psi_{i}: I \times I \rightarrow$ $E_{i}^{*} \times I$ ending at $\bar{\gamma}_{i} \times\{1\}$ so that $\operatorname{im}\left(\psi_{i}\right) \cap \operatorname{im}\left(\psi_{j}\right)=\emptyset$ if $i \neq j$.

To establish the appropriate control for the replacement procedure, choose $\xi$ so that

- $0<\xi<\varepsilon$,

- $d\left(\left\{x_{1}, \ldots, x_{m}\right\},\left\{y_{1}, \ldots, y_{m}\right\}\right)>\xi$ in $K$

- $N\left(\operatorname{im}\left(\psi_{i}\right) ; \xi\right) \subset E_{i}^{*} \times I$,

- $N\left(\operatorname{im}\left(\psi_{i}\right) ; \xi\right) \cap N\left(\operatorname{im}\left(\psi_{j}\right) ; \xi\right)=\emptyset$ for $i \neq j$,

- $N\left(\bar{\gamma}_{i} ; \xi\right) \subset G_{<\varepsilon}$

- $\pi\left(N\left(\bar{\gamma}_{i} ; \xi\right)\right) \cap \pi\left(N\left(\bar{\gamma}_{j} ; \xi\right)\right)=\emptyset$ whenever $i \neq j$.

Note that the last two conditions are possible since $\lambda^{*} \cup \bigcup_{i=1}^{m} \gamma_{i} \in \mathcal{A}^{*}$. Now let $Y^{*}=\bigcup_{i=1}^{m} N\left(\operatorname{im}\left(\psi_{i}\right) ; \xi\right)$ and $K^{*}$ be the complex obtained by identifying each $x_{i}$ to $y_{i}$ in $K$. 
By Corollary 10.3, there exist a finite set $\left\{D_{1}, \ldots, D_{m}\right\}$ of disjoint p.l. disks in $K$ and an isotopy $H^{\prime}: K \times I \rightarrow M$ so that

(1) $x_{i} \in D_{i}$,

(2) $\operatorname{diam}\left(D_{i}\right)<\xi$

(3) $\widetilde{H}^{\prime}\left(D_{i} \times I\right) \subset N\left(\operatorname{im}\left(\psi_{i}\right) ; \xi\right)$,

(4) $H^{\prime}=H$ on $\overline{K-\bigcup_{i=1}^{m} D_{i}} \times I$,

(5) $H_{0}^{\prime}=H_{0}$.

Note that $H^{\prime}$ is a $\left(3 \zeta, L^{\prime}\right)[\pi]$-isotopy and hence a $\left(\delta, L^{\prime}\right)[\pi]$-isotopy.

By Theorem 11.1 and our choices of $\zeta$ and $\delta$, there is a cover $\Gamma$ of $H^{\prime}$ that is an $\varepsilon[\pi]$-push of $f^{*}\left(\overline{K-L^{\prime}} \times I\right)$ supported in $U^{*}$.

Now we verify that $\Gamma$ is the desired isotopy.

(1) $\Gamma$ is supported in $U^{*}$ by construction.

(2) Since $f^{*}=H_{0}=H_{0}^{\prime}$ and $H^{\prime}=\Gamma \circ\left(H_{0}^{\prime} \times\right.$ id $)$, we have $\Gamma \circ\left(f^{*} \times\right.$ id $)=$ $H^{\prime}$. Note that $H^{\prime}\left(D_{i} \times I\right) \subset E_{i}^{*}$ and $\operatorname{diam}\left(E_{i}^{*}\right)<4 \eta$. Furthermore, $H^{\prime}=H$ away from $\bigcup_{i=1}^{m} D_{i} \times I$ and $H$ is a $\left(2 \eta, L^{\prime}\right)$-isotopy. It follows that $H^{\prime}$ is a $\left(4 \eta, L^{\prime}\right)$-isotopy. Hence $\Gamma \circ\left(f^{*} \times\right.$ id $)$ is a $\left(4 \eta, L^{\prime}\right)$-isotopy.

(3) By construction $\Gamma$ is an $\varepsilon[\pi]$-isotopy.

(4) $\Gamma$ pushes $f^{*}(K)$ into $G_{<\varepsilon}$ as a result of the choice of $\xi$.

(5) $\operatorname{diam}(D)<\xi<\varepsilon$ for $D \in \mathcal{D}$.

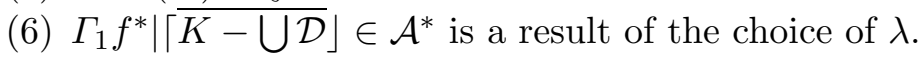

(7) Since $\Gamma_{1} f^{*}$ is an embedding on $K \times I$ and $\pi \Gamma_{1} f^{*}$ is an embedding on $\overline{K-\bigcup \mathcal{D}} \times I$, by (6) and the choice of $\xi$ it follows that for each $D_{i} \in \mathcal{D}, \pi \Gamma_{1} f^{*}\left(D_{i}\right) \cap \pi \Gamma_{1} f^{*}\left(K-D_{i}\right)=\emptyset$.

(8) It follows from the choice of $E_{i}^{*}$ that $\operatorname{diam}\left(\Gamma_{1} f^{*}\left(D_{i}\right)\right)<4 \eta$ for $i=$ $1, \ldots, m$.

Therefore $\Gamma$ is the desired isotopy.

The proof of the "moreover" part of this theorem follows from the application of the "moreover" conditions of the theorems of Section 10. Let $Z$ be a subset of $M$ missing $f^{*}(K)$ such that $\pi(Z)$ is $\operatorname{LCC}^{1}$ in $M / G$. Without loss of generality, we may assume that the artifact maps are chosen so that the projection of the initial ends $\pi\left(\bigcup_{i=1}^{m} \Pi_{M}\left(\left(\psi_{i}\right)_{0}\right)\right)$ misses $\pi(Z)$. Choose $\phi: M \times I \rightarrow M \times I$ to be an $(H, \xi)$-buttressing map where $\xi$ is chosen as before. Since on each level $M \times\{t\}, \phi$ collapses an arc not bounding a simplex in $\widetilde{H}(K \times I)$, we may select $\phi$ so that $\phi H$ is p.l. on $K \times\left\{0, t_{1}, \ldots, t_{m}, 1\right\}$. By Theorem 9.10, there is a p.l. isotopy $J: K^{*} \times I \rightarrow M$ that is $N\left(\bigcup_{i=1}^{m} \operatorname{im}\left(\psi_{i}\right) ; \xi\right)$ near $H$ so that $J \circ(\theta \times$ id $)=H$ on $\overline{K-L^{\prime}} \times I$ and $\widetilde{J} \circ(\theta \times$ id $)=\phi \widetilde{H}$ on $K \times\left\{0, t_{1}, \ldots, t_{m}, 1\right\}$. Note that $J$ is a $(\delta, L)[\pi]$-isotopy. By Theorem 11.1, there is an $\varepsilon[\pi]$-push $\Lambda: M \times I \rightarrow M$ of $f^{*}\left(\overline{K-L^{\prime}}\right)$ supported in $Y^{*}=$ $\bigcup_{i=1}^{m} N\left(\operatorname{im}\left(\psi_{i}\right) ; \xi\right)$ that is a cover of $J$. Let $A=\pi^{-1} \pi\left(\Pi_{M} \phi^{-1} \widetilde{\Lambda}(Z \times\{1\})\right)$. 
Since the ends of the artifact maps $\psi_{i}$ are contained in the nondegeneracy set of $G \times\{1\}$ and $(Z \times\{0\}) \cap \bigcup_{i=1}^{m} \psi_{i}(I \times\{0\})=\emptyset$, we have $(A \times\{1\}) \cap \bigcup_{i=1}^{m} \psi_{i}(I \times\{1\})=\emptyset$. The problem is, when we do the replacement procedures, as outlined in the proof of Corollary 10.3, if we are not careful, $A$ may meet the images $H_{1}^{\prime}\left(D_{i}\right)$, thereby causing failure of the "moreover" condition. To address this problem, let $\Omega$ be an open set in $M \times I$ containing $\bigcup_{i=1}^{m} \operatorname{im}\left(\psi_{i}\right)$ and missing $A \times\{1\}$. For convenience, we also require that $\Omega \cap(M \times\{1\})$ is a $(G \times\{1\})$-saturated open set in $M \times\{1\}$. Now when applying Corollary 10.3, we require, in addition to the five previously required conditions, that

(6) $H^{\prime}$ is covered by an isotopy $\Gamma: M \times I \rightarrow M$ respecting the singularities of $\widetilde{H}$ so that $\widetilde{\Gamma} \widetilde{\Lambda}^{-1}=$ id on $\left[(M \times I)-N\left(\bigcup \operatorname{im}\left(\psi_{i}\right) ; \xi\right)\right] \cup$ $\bigcup_{i=1}^{m}\left(N\left(\psi_{i} ; \xi\right) \cap\left(M \times\left\{t_{i}\right\}\right)\right)$ and $\widetilde{\Gamma} \widetilde{\Lambda}^{-1} \phi=\mathrm{id}$ on $(M \times I)-\Omega$.

We now desire to show that

$$
\pi \Gamma_{1}(Z) \cap \pi \Gamma_{1} f^{*}(K)=\emptyset .
$$

It suffices to prove that

$$
\left[\pi^{-1} \pi \Gamma_{1}(Z) \times\{1\}\right] \cap\left[\pi^{-1} \pi \Gamma_{1} f^{*}(K) \times\{1\}\right]=\emptyset .
$$

Note that

$$
\pi^{-1} \pi \Gamma_{1}(Z)=\pi^{-1} \pi \Pi_{M} \widetilde{\Gamma}(Z \times\{1\})=\pi^{-1} \pi \Pi_{M} \phi^{-1} \widetilde{\Lambda}(Z \times\{1\})=A .
$$

From the details of proofs of Theorems 10.1 and 10.2 and our choice of $\Omega$, we have

$$
\Gamma_{1} f^{*}\left(\bigcup_{i=1}^{m} D_{i}\right) \times\{1\} \subset \Omega
$$

Thus

$$
\left[\pi^{-1} \pi \Gamma_{1}(Z) \times\{1\}\right] \cap\left[\pi^{-1} \pi \Gamma_{1} f^{*}\left(\bigcup_{i=1}^{m} D_{i}\right) \times\{1\}\right] \subset(A \times\{1\}) \cap \Omega=\emptyset .
$$

Also $Z \cap f^{*}(K)=\emptyset$ so $\Gamma_{1}(Z) \cap \Gamma_{1} f^{*}(K)=\emptyset$. Since $\Gamma_{1} f^{*}\left(K-\bigcup_{i=1}^{m} D_{i}\right) \subset N_{G}$,

$$
\left[\pi^{-1} \pi \Gamma_{1}(Z) \times\{1\}\right] \cap\left[\pi^{-1} \pi \Gamma_{1} f^{*}\left(K-\bigcup_{i=1}^{m} D_{i}\right) \times\{1\}\right]=\emptyset .
$$

Therefore

$$
\left[\pi^{-1} \pi \Gamma_{1}(Z) \times\{1\}\right] \cap\left[\pi^{-1} \pi \Gamma_{1} f^{*}(K) \times\{1\}\right]=\emptyset .
$$

We conclude that

$$
\pi \Gamma_{1}(Z) \cap \pi \Gamma_{1} f^{*}(K)=\emptyset
$$

so the "moreover" condition is satisfied. 
We need just one more result which, in combination with Theorem 11.2, ensures that $K$ can be pushed into $N_{G}^{c}$.

Proposition 11.3. Let $K$ be a finite 2-complex and $M$ a 5-manifold. Suppose for $i=1,2, \ldots$ that:

(1) The set $\mathcal{D}_{i}$ consists of $m_{i}$ pairwise disjoint p.l. disks in $K$ so that

(a) for each $D \in \mathcal{D}_{i+1}$ there is a $D^{\prime} \in \mathcal{D}_{i}$ so that $D \subset \operatorname{int}\left(D^{\prime}\right)$ (in this case we denote $D^{\prime}$ as $\left.\operatorname{pre}(D)\right)$,

(b) $\lim _{i \rightarrow \infty} \max \left\{\operatorname{diam}(D) \mid D \in \mathcal{D}_{i}\right\}=0$.

(2) There is a sequence of homeomorphisms $\lambda_{i}: K \rightarrow M$ such that $\lambda_{i+k}\left|\left\lceil\overline{K-\bigcup \mathcal{D}_{i}}\right\rfloor=\lambda_{i}\right|\left\lceil\overline{K-\bigcup \mathcal{D}_{i}}\right\rfloor$ for all $k>0$.

(3) The set $\mathcal{U}_{i}$ consists of $m_{i}$ pairwise disjoint open sets such that

(a) each $D \in \mathcal{D}_{i}$ corresponds to a unique $U \in \mathcal{U}_{i}$ such that $\lambda_{i}(D) \cup$ $\lambda_{i+1}(D) \subset U$ and $\lambda_{i}^{-1}(U) \subset \operatorname{pre}(D)$,

(b) $\bigcup \mathcal{U}_{1} \supset \overline{\cup \mathcal{U}_{2}} \supset \bigcup \mathcal{U}_{2} \supset \overline{\cup \mathcal{U}_{3}} \supset \cdots$,

(c) $\lim _{i \rightarrow \infty} \max \left\{\operatorname{diam}(U) \mid U \in \mathcal{U}_{i}\right\}=0$.

Then $\lambda=\lim _{i \rightarrow \infty} \lambda_{i}$ is an embedding of $K$.

Proof. It is clear from (2) and (3) that $\lambda=\lim _{i \rightarrow \infty} \lambda_{i}$ exists and is continuous. To see that $\lambda$ is $1-1$, let $x, y \in K$. Let $Z=\bigcap_{i=1}^{\infty}\left(\bigcup \mathcal{D}_{i}\right)$. There are three cases to consider:

Case 1: $x, y \in K-Z$. There is some $i$ so that $x, y \in K-\bigcup \mathcal{D}_{i}$. By (2), $\lambda(x) \neq \lambda(y)$.

CASE 2: $x \in K-Z$ and $y \in Z$ or $y \in K-Z$ and $x \in Z$. Without loss of generality let $x \in K-Z$ and $y \in Z$. From (1a), there exists an $N$ so that $x \notin \bigcup \mathcal{D}_{i}$ for all $i \geq N$. From (3a), $\lambda_{i+1}^{-1}\left(\bigcup \mathcal{U}_{i+1}\right) \subset \bigcup \mathcal{D}_{i}$. Hence $\lambda_{i+1}(x) \notin \bigcup \mathcal{U}_{i+1}$ for all $i \geq N$. By $(2), \lambda_{i}(x)=\lambda(x)$ for all $i \geq N$. It is then clear from (3b) that $\lambda(x) \notin \bigcap_{i=1}^{\infty}\left(\cup \mathcal{U}_{i}\right)$. On the other hand, it follows from (3a) and (3b) that $\lambda(y) \in \bigcap_{i=1}^{\infty}\left(\bigcup \mathcal{U}_{i}\right)$. Therefore $\lambda(x) \neq \lambda(y)$.

CASE 3: $y \in Z$ and $x \in Z$. By (1b) there is an $i$ so that there are disjoint balls $D_{x}, D_{y} \in \mathcal{D}_{i}$ such that $x \in D_{x}$ and $y \in D_{y}$. It follows from (3a) and (3b) that there are disjoint open sets $U_{x}, U_{y} \in \mathcal{U}_{i+1}$ such that $\lambda_{i+1}(x) \in U_{x}$, $\lambda_{i+1}(y) \in U_{y}$ and $\bar{U}_{x} \cap \bar{U}_{y}=\emptyset$. It is also clear from (3a) and (3b) that $\lambda_{i+k}(x) \in \bar{U}_{1}$ and $\lambda_{i+k}(y) \in \bar{U}_{y}$ for all $k \geq 1$. It follows that $\lambda(x) \in \bar{U}_{x}$ and $\lambda(y) \in \bar{U}_{y}$. Since $\bar{U}_{x} \cap \bar{U}_{y}=\emptyset$, we conclude that $\lambda(x) \neq \lambda(y)$.

Therefore $\lambda$ is $1-1$ and gives an embedding of $K$.

The following corollary is the main result for this section.

Corollary 11.4. Let $G$ be a cell-like usc decomposition of a 5-manifold $M$ for which $\operatorname{dim}(M / G)<\infty$ and the associated decomposition map $\pi$ : 
$M \rightarrow M / G$ is $L C C^{1}$ refined over $\mathcal{A}$. Suppose that $K$ is a finite 2 -complex and $f: K \rightarrow M$ is an $L C C^{1}$ embedding. Then for any $\varepsilon>0$, there is a cell-like map $h: M \rightarrow M$ that is the end of an $\varepsilon[\pi]$-pseudo-isotopy such that $h \mid\lceil f(K)\rfloor$ is an $L C C^{1}$ embedding, $h f(K) \cap N_{G}=\emptyset, h\left(N_{h}\right) \subset h f(K)$, and $\operatorname{dem}\left(N_{h}\right) \leq 2$. Moreover, if $\Omega$ is a $G$-saturated neighborhood of $f(K)$ we may assume that $h$ is supported in $\Omega$.

Proof. Let $\varepsilon>0$ be given. Using Theorem 11.2, we will define $h$ as the limit of a sequence of pushes of $f(K)$ whose restrictions to $f(K)$ satisfy the hypotheses of Proposition 11.3.

In order to ensure that the end of the pending pseudo-isotopy, which will be denoted as $h$, has the property that $h f(K)$ is $\operatorname{LCC}^{1}$ and $\operatorname{dem}\left(N_{h}\right) \leq 2$, we identify a sequence of triangulations of $M$, namely $T_{1}, T_{2}, \ldots$, such that

- $\operatorname{mesh}\left(T_{i}\right) \rightarrow 0$,

- the 2-skeleton $T_{i}^{(2)}$ of each $T_{i}$ misses $f(K) \cup \bigcup\left\{\operatorname{im}\left(\alpha_{i}^{*}\right) \mid \alpha_{i}^{*} \in \mathcal{A}^{*}\right\}$. This condition is possible because $f(K)$ is an $\mathrm{LCC}^{1}$ embedding and because $\pi$ is $\mathrm{LCC}^{1}$ refined over $\mathcal{A}$.

We will implement controls in the construction of the desired pseudo-isotopy so that $h f(K)$ and $N_{h}$ miss $\bigcup_{i=1}^{\infty} T_{i}$. It will follow that the end of the pseudoisotopy will take $f(K)$ to an $\mathrm{LCC}^{1}$ embedding and that $\operatorname{dem}\left(N_{h}\right) \leq 2$.

Let $Z_{i}=\pi^{-1} \pi\left(\bigcup_{j=1}^{i} T_{j}^{(2)}\right)$. By our choice of $T_{i}$ and since $\pi$ is $\mathrm{LCC}^{1}$ refined over $\mathcal{A}$, each $\pi\left(Z_{i}\right)$ misses the set $\bigcup\left\{\operatorname{im}\left(\alpha_{i}\right) \mid \alpha_{i} \in \mathcal{A}\right\}$. Hence $\pi\left(Z_{i}\right)$ is $\mathrm{LCC}^{1}$.

STEP 1: The first push. Choose $\eta_{1}$ sufficiently large so that $f(K) \subset G_{\eta_{1}}$. Let $\varepsilon_{1}=\min \left\{\varepsilon / 4, \eta_{1}\right\}, L_{1}=L_{1}^{\prime}=\emptyset$ and $U_{1}=\Omega$. By Theorem 11.2 there is a set $\mathcal{D}_{1}$ consisting of disjoint p.l. disks in $K-L_{1}$ and an isotopy $\Gamma^{1}$ : $M \times I \rightarrow M$ so that

(1) $\Gamma^{1}$ is supported in $U_{1}$,

(2) $\Gamma^{1} \circ(f \times$ id $)$ is a $\left(4 \eta_{1}, L_{1}^{\prime}\right)$-isotopy,

(3) $\Gamma^{1}$ is also an $\varepsilon_{1}[\pi]$-isotopy,

(4) $\Gamma^{1}$ pushes $f(K)$ into $G_{<\varepsilon_{1}}$,

(5) for $D \in \mathcal{D}_{1}, \operatorname{diam}(D)<\varepsilon_{1}$,

(6) $\Gamma_{1}^{1} f \mid\left\lceil\overline{K-\bigcup \mathcal{D}_{1}}\right\rfloor \in \mathcal{A}^{*}$

(7) if $D \in \mathcal{D}_{1}$, then $\pi \Gamma_{1}^{1} f(D) \cap \pi \Gamma_{1}^{1} f(K-D)=\emptyset$,

(8) for all $D \in \mathcal{D}_{1}$, $\operatorname{diam}\left(\Gamma_{1}^{1} f(D)\right)<4 \eta_{1}$,

(9) $\pi \Gamma_{1}^{1}\left(Z_{1}\right) \cap \pi \Gamma_{1}^{1} f(K)=\emptyset$.

Define $h_{1}=\Gamma_{1}^{1}$ and $\lambda_{1}=\Gamma_{1}^{1} f$.

STEP 2: The ith push for $i>1$. Suppose that $\varepsilon_{i-1}, \lambda_{i-1}$, and $\mathcal{D}_{i-1}$ are given. Let $\eta_{i}=\varepsilon_{i-1}$. Note that $\lambda_{i-1}(K) \subset G_{\eta_{i}}$. Define $\varepsilon_{i}=\min \left\{\varepsilon / 2^{i+1}, \eta_{i}\right\}$ 
and $L_{i}^{\prime}=\overline{K-\bigcup \mathcal{D}_{i-1}}$. Let $\mathcal{D}_{i-1}^{\prime}$ be a collection of disjoint disk neighborhoods of the elements of $\mathcal{D}_{i-1}$ in some $k_{i}$ th barycentric subdivision of $K$ where $k_{i}$ is chosen sufficiently large so that the disks in $\mathcal{D}_{i-1}^{\prime}$ satisfy conditions (5) through (8) listed above when $\mathcal{D}_{i-1}^{\prime}$ is replaced with $\mathcal{D}_{i-1}$. Let $L_{i}=\overline{K-\bigcup \mathcal{D}_{i-1}^{\prime}}$.

Now let $\mathcal{U}_{i}$ denote a collection of $G$-saturated neighborhoods of the elements of $\left\{\lambda_{i-1}(D) \mid D \in \mathcal{D}_{i}\right\}$, one neighborhood for each disk in $\mathcal{D}_{i}$, so that $\bar{\cup} \mathcal{U}_{i} \subset \bigcup \mathcal{U}_{i-1}$. Let $U_{i}=\bigcup \mathcal{U}_{i}$. From condition (7) and the choice of $\mathcal{D}_{i}^{\prime}$, we may assume that these neighborhoods are pairwise disjoint and if $U$ is an element of $\mathcal{U}_{i}$ containing $D \in \mathcal{D}_{i}$, and $D^{\prime} \in \mathcal{D}_{i}^{\prime}$ is the disk such that $D \subset D^{\prime}$, then $\lambda_{i-1}^{-1}(U) \cap K \subset \operatorname{int}\left(D^{\prime}\right)$. We impose the following conditions on $\mathcal{U}_{i}$ :

- $\pi\left(U_{i}\right) \cap \pi h_{i-1}\left(Z_{i-1}\right)=\emptyset$ (see condition (9)),

- $\pi\left(U_{i}\right) \cap \pi\left(Z_{i}\right)=\emptyset$ (from the choice of $Z_{i}$ ), and

- if $U \in \mathcal{U}_{i}$ then $\operatorname{diam}(U)<4 \eta_{i-1}+2 \varepsilon_{i-1}<6 \eta_{i}$ (see conditions (4) and (8)).

By Theorem 11.2 there is a set $\mathcal{D}_{i}$ consisting of disjoint p.l. disks in $K-L_{i}$ and an isotopy $\Gamma^{i}: M \times I \rightarrow M$ so that

(1) $\Gamma^{i}$ is supported in $U_{i}$,

(2) $\Gamma^{i} \circ\left(\lambda_{i-1} \times\right.$ id $)$ is a $\left(4 \eta_{1}, L_{i}^{\prime}\right)$-isotopy,

(3) $\Gamma^{i}$ is also an $\varepsilon_{i}[\pi]$-isotopy,

(4) $\Gamma^{i}$ pushes $\lambda_{i-1}(K)$ into $G_{<\varepsilon_{i}}$,

(5) for $D \in \mathcal{D}_{i}, \operatorname{diam}(D)<\varepsilon_{i}$,

(6) $\Gamma_{1}^{i} \lambda_{i-1} \mid\left\lceil\overline{K-\bigcup \mathcal{D}_{i}}\right\rfloor \in \mathcal{A}^{*}$.

(7) if $D \in \mathcal{D}_{i}$, then $\pi \Gamma_{1}^{i} \lambda_{i-1}(D) \cap \pi \Gamma_{1}^{i} \lambda_{i-1}(K-D)=\emptyset$,

(8) for all $D \in \mathcal{D}_{i}, \operatorname{diam}\left(\Gamma_{1}^{i} \lambda_{i-1}(D)\right)<4 \eta$,

(9) $\pi \Gamma_{1}^{i}\left(Z_{i}\right) \cap \pi \Gamma_{1}^{i} f(K)=\emptyset$.

Define $h_{i}=\Gamma_{1}^{i} \circ \Gamma_{1}^{i-1} \circ \cdots \circ \Gamma_{1}^{1}$ and $\lambda_{i}=\Gamma_{1}^{i} \lambda_{i-1}$. Note that

- The set $\mathcal{D}_{i}$ consists of a finite number, say $m_{i}$, of disjoint p.l. disks in $K$ so that

(a) for each $D \in \mathcal{D}_{i+1}$ there is a $D^{\prime} \in \mathcal{D}_{i}$ so that $D \subset \operatorname{int}\left(D^{\prime}\right)$,

(b) $\lim _{i \rightarrow \infty} \max \left\{\operatorname{diam}(D) \mid D \in \mathcal{D}_{i}\right\} \leq \lim _{i \rightarrow \infty} \varepsilon_{i}=0$.

- The sequence of homeomorphisms $\lambda_{i}: K \rightarrow M$ satisfies $\lambda_{i+k} \mid\left\lceil\overline{K-\bigcup \mathcal{D}_{i}}\right\rfloor$ $=\lambda_{i} \mid\left\lceil\overline{K-\bigcup \mathcal{D}_{i}}\right\rfloor$ for all $k>0$.

- The set $\mathcal{U}_{i}$ consists of $m_{i}$ disjoint open sets such that

(a) each $D \in \mathcal{D}_{i}$ corresponds to a $U \in \mathcal{U}_{i}$ such that $\lambda_{i}(D) \cup \lambda_{i+1}(D)$ $\subset U$ and $\lambda_{i}^{-1}(U) \subset \operatorname{pre}(D)$,

(b) $\bigcup \mathcal{U}_{1} \supset \overline{\overline{U \mathcal{U}}_{2}} \supset \bigcup \mathcal{U}_{2} \supset \overline{\mathcal{U U}_{3}} \supset \cdots$,

(c) $\lim _{i \rightarrow \infty} \max \left\{\operatorname{diam}(U) \mid U \in \mathcal{U}_{i}\right\} \leq \lim _{i \rightarrow \infty} 6 \eta_{i}=0$. 
Let

$$
h=\lim _{i \rightarrow \infty} h_{i} \quad \text { and } \quad \lambda=\lim _{i \rightarrow \infty} \lambda_{i} .
$$

Note that $h$ is the end of the pseudo-isotopy $\Gamma=\lim _{i \rightarrow \infty} \Gamma^{i} * \Gamma^{i-1} * \cdots * \Gamma^{1}$. By condition (3) of the construction of $\Gamma^{i}, \Gamma$ is an $\varepsilon[\pi]$-pseudo-isotopy. It follows from Proposition 11.3 that $\lambda=h f$ is an embedding. Since $h f(K) \subset$ $G_{<\eta_{i}}$ for all $i$, we have $h f(K) \subset N_{G}^{c}$.

To see that $h\left(N_{h}\right) \subset h f(K)$, we first show that $\bigcap_{i=1}^{\infty} \overline{\cup \mathcal{U}_{i}} \subset h(K)$. Suppose that $y \in \bigcap_{i=1}^{\infty} \overline{\cup \mathcal{U}_{i}}$. Then for each $i$ there is an open set $U_{i} \in$ $\mathcal{U}_{i}$ so that $y \in \bar{U}_{i}$. Since $\operatorname{diam}\left(U_{i}\right)<6 \eta_{i}$, it follows that $\bigcap_{i=1}^{\infty} \bar{U}_{i}=y$. Also for each $i$ there is a disk $D_{i} \subset K$ so that $h_{i} f(K)=\lambda_{i}\left(D_{i}\right) \subset U_{i}$, $D_{i+1} \subset D_{i}$, and $\bar{U}_{i+1} \subset U_{i}$. Let $x \in \bigcap_{i=1}^{\infty} D_{i}$. Then $h_{i} f(x) \subset U_{i}$ for all $i$. Hence $h f(x) \in \bigcap_{i=1}^{\infty} \bar{U}_{i}$ so $h f(x)=y$. Therefore $h\left(N_{h}\right) \subset h f(K)$. Now suppose that $h(p)=h(q) \in h(K)$. Then for some $i, h(p)=h(q) \notin \bar{U}_{i}$. But then by construction, $h(p)=h_{i}(p) \neq h_{i}(q)=h(q)$, which contradicts our supposition. Therefore, $h\left(N_{h}\right) \subset h f(K)$.

We claim that $h f(K)$ misses $\bigcup_{i=1}^{\infty} Z_{i}$. If $x \in K-\bigcap_{i=1}^{\infty}\left(\bigcup \mathcal{D}_{i}\right)$ then there is an $i$ so that $x \in K-\bigcup \mathcal{D}_{i}$. It follows that $h f(x)=\lambda_{i}(x) \notin \bigcup_{i=1}^{\infty} Z_{i}$. If $x \in \bigcap_{i=1}^{\infty}\left(\cup \mathcal{D}_{i}\right)$, then for all $i, h f(x) \in U_{i}$ which misses $Z_{i}$. Again it follows that $f h(x) \notin \bigcup_{i=1}^{\infty} Z_{i}$. Therefore, for all $x \in K, f h(x) \notin \bigcup_{i=1}^{\infty} Z_{i}$. Hence $h f(K)$ misses $\bigcup_{i=1}^{\infty} T_{i}^{(2)}$, which ensures that $h \mid\lceil f(K)\rfloor$ is an $\mathrm{LCC}^{1}$ embedding.

We also claim that $N_{h}$ misses $\bigcup_{i=1}^{\infty} Z_{i}$. If $p \notin \bigcap_{i=1}^{\infty} U_{i}$, then there is a $j$ so that $p \notin U_{i}$ for all $i \geq j$. If $q \in Z_{k}$ and $m=\max \{j, k\}$, then $h(p)=h_{m}(p) \neq h_{m}(q)=h(q)$. Suppose $p \in \bigcap_{i=1}^{\infty} U_{i}$ and $q \in Z_{k}$. Then $h(p) \in \bigcap_{i=1}^{\infty} \bar{U}_{i}=\bigcap_{i=1}^{\infty} U_{i}$. However, $h(q)=h_{k}(q) \notin U_{k}$. Thus $\bigcup_{i=1}^{\infty} Z_{i}$ does not meet $N_{h}$. It follows that $N_{h}$ misses $\bigcup_{i=1}^{\infty} T_{i}^{(2)}$, which ensures that $\operatorname{dem}\left(N_{h}\right) \leq 2$. Clearly, $h$ is supported in $\Omega$. Therefore $h$ is the desired map.

12. Shrinking fixing a complex. In this section we will describe the technology for shrinking a decomposition while fixing a 2-complex $K$. The symbol $M$ will be used throughout to denote a compact p.l. 5-manifold. The notation $\pi$ will denote an arbitrary decomposition map, and is not intended to represent the decomposition map specified in the previous section. The purpose is to establish a procedure by which we can shrink out the decomposition of $M$ induced by the map $h$ given in the conclusion of Corollary 11.4 without perturbing the complex $K$. In particular, this section is devoted to proving the following theorem:

Theorem 12.1. Suppose $K$ is a finite 2-complex $L C C^{1}$ embedded in $M$, and $G$ is a cell-like usc decomposition of $M$ for which the decomposition map $\pi: M \rightarrow M / G$ is 1-1 on $K, N_{G} \subset \pi^{-1} \pi(K)$ and $\operatorname{dem}\left(N_{G}\right) \leq 2$. 
Then for each $\varepsilon>0$ and each neighborhood $U$ of $N_{G}$ in $M$ there exists a homeomorphism $F: M \rightarrow M / G$ such that

(1) $F|\lceil K\rfloor=\pi|\lceil K\rfloor$,

(2) $F|\lceil M-U\rfloor=\pi|\lceil M-U\rfloor$,

(3) $\varrho(F, \pi)<\varepsilon$.

The proof of this theorem will follow from standard shrinking technology, as developed by Edwards [16], modified to perform that shrinking while fixing a preassigned subset.

Definition 12.2. Suppose $S$ is a metric space, $K$ is a subset of $S, G$ is a usc decomposition of $S$, and $\pi: S \rightarrow X$ is the associated decomposition map. Then $G$ is strongly shrinkable fixing $K$ provided that for every $\varepsilon>0$ and every neighborhood $U$ of $N_{G}$, there is a homeomorphism $\Phi: S \rightarrow S$ satisfying

(1) $\Phi|\lceil K\rfloor=\mathrm{id}|\lceil K\rfloor$,

(2) $\Phi$ is supported in $U$,

(3) $\operatorname{diam}(\Phi(g))<\varepsilon$ for all $g \in G$,

(4) $\varrho(\pi, \pi \Phi)<\varepsilon$.

Note that if $G$ is strongly shrinkable fixing $K$, then $\pi$ is necessarily 1-1 on $K$.

The proofs of the next two propositions are exactly the same as those found in [11, Theorem 5.2] and [11, Corollary 5.2D], respectively, with added stipulations that the relevant maps and homeomorphisms fix $K$.

Proposition 12.3. Suppose $G$ is a usc decomposition of a compact metric space $S$ and $K$ is a closed subset of $S$. Then the decomposition map $\pi: S \rightarrow S / G$ can be approximated, arbitrarily closely, by homeomorphisms fixing $K$ and supported in any preassigned neighborhood $U$ of $N_{G}$ if and only if $G$ is strongly shrinkable fixing $K$.

Proposition 12.4. Suppose $G$ is a usc decomposition of a compact metric space $S$ with associated decomposition map $\pi: S \rightarrow S / G$, and $K$ is a closed subset of $S$. Then $G$ is strongly shrinkable fixing $K$ if and only if, for each $\varepsilon>0$ and each neighborhood $U$ of $N_{G}$, there exists a map $\mu: S \rightarrow S$ such that

- $\mu|\lceil K\rfloor=\mathrm{id}|\lceil K\rfloor$,

- $\mu$ is supported in $U$,

- $G=\left\{\mu^{-1}(s) \mid s \in S\right\}$,

- $\varrho\left(\pi \mu^{-1}, \pi\right)<\varepsilon$.

Proposition 12.5. Suppose $K$ is a finite 2-complex $L C C^{1}$ embedded in $M, C$ is a cell-like set such that $\operatorname{dem}(C) \leq 2$ and $C \cap K$ is cell-like, $\varepsilon>0$, and $D$ is a topological cone neighborhood of $C \cap K$ in $K$ such that 
$C \cap K \subset \operatorname{int}(D) \subset D \subset N(C \cap K ; \varepsilon)$. Then there exists a 5-cell $B$ in $M$ such that $C \subset \operatorname{int}(B) \subset B \subset N(C ; \varepsilon)$ and $B \cap K=D$, and $D$ is standardly embedded (i.e., equivalent to a subcone of $B \cong O * S^{4}$ ) in $B$.

Proof. Treat $K$ as a subcomplex of $M$ [5]. Start with a 5-cell $E$ that is a combinatorial cone neighborhood in $M$ of some point $p \in C \cap K$, where $E \cap K=D$ is a subcone of $E$ (for instance, when $D$ is a 2-cell then $E \cong$ $B^{2} \times B^{3}$ with $\left.D=B^{2} \times 0 \subset B^{2} \times B^{3}\right)$ and $E \subset N(C ; \varepsilon)$. Standard engulfing techniques give a homeomorphism $\Theta: M \rightarrow M$ fixing $K \cup(M-N(C ; \varepsilon))$ such that, for $B=\Theta(E), C \subset \operatorname{int}(B)$.

Here is an outline of how the engulfing works. Specify a triangulation $T$ of $M$ having very small mesh, with $K$ carried by the 2-skeleton of $T$. Let $V$ be a compact subpolyhedron carried by a subcomplex of $T$, with $C \subset \operatorname{int}(V) \subset V \subset N(C ; \varepsilon)$, and let $P$ denote the 2-skeleton of $V$ relative to $T$. With appropriate size controls, $V$ can be chosen so that there exists an engulfing homeomorphism $\theta_{1}: M \rightarrow M$ fixed outside $N(C ; \varepsilon)$ and on $K$ such that $P \subset \theta_{1}(\operatorname{int}(E))$. In the first barycentric subdivision $V^{\prime}$ of the simplicial structure on $V$ inherited from $T$, let $Q$ denote the dual 2-skeleton to $P$. Since $C \cap K$ misses $Q$ and $\operatorname{dem}(C) \leq 2$, there exists another homeomorphism $\theta_{2}$ : $M \rightarrow M$ (moving points very little) which is fixed on $K \cup(M-V)$ and moves $C$ off $Q$, achieving $Q \subset \theta_{2}(M-C)$. Finally, stretch across the join structure of $V^{\prime}$ via a third homeomorphism $\theta_{3}: M \rightarrow M$, fixing $P \cup Q \cup(M-V)$, such that

$$
\theta_{3} \theta_{1}(\operatorname{int}(E)) \cup \theta_{2}(M-C)=M .
$$

Set $\Theta=\theta_{2}^{-1} \theta_{3} \theta_{1}$, and note that $\Theta$ fixes $K$. Applying $\theta_{2}^{-1}$ to the above equality, we find

$$
\Theta(\operatorname{int}(E)) \cup(M-C)=\theta_{2}^{-1}(M)=M,
$$

so clearly $C \subset \Theta(\operatorname{int}(E))=\operatorname{int}(B)$, and $B \subset N(C ; \varepsilon)$, since $\Theta$ restricts to the identity outside $N(C ; \varepsilon)$.

Lemma 12.6. Consider $B^{n}$ as a cone $O * \partial B^{n}$. Suppose $Z$ is a compact set in $\partial B^{n}$ and $Y$ is a compact set in $\operatorname{int}\left(B^{n}\right)$. Then for any $\varepsilon>0$ there is a homeomorphism $\theta: B^{n} \rightarrow B^{n}$ fixing $\partial B^{n}$ and $O * Z$ such that $\psi(Y)$ is contained in the $\varepsilon$-neighborhood of $O * Z$.

Proof. It suffices to prove this proposition in the case of $B^{n}=\left\{\mathbf{x} \in \mathbb{R}^{n} \mid\right.$ $\|\mathbf{x}\|<1\}$ where $O$ is the origin $\mathbf{0}$. Choose $\gamma>0$ so that $Y \subset \operatorname{int}\left(\gamma B^{n}\right)$. Assume without loss of generality that $\varepsilon<\gamma$ and obtain a continuous function $\tau: \partial B^{n} \rightarrow[\varepsilon, \gamma]$ so that $\tau(Z)=\gamma$ and $\tau\left(\partial B^{n}-N(Z, \varepsilon)\right)=\varepsilon$. Define a homeomorphism $\psi: B^{n} \rightarrow B^{n}$ such that $\psi$ is the identity map on $\partial B^{n} \cup\{\mathbf{0}\}$, $\psi(\mathbf{x})=(\tau(\mathbf{x}) / \gamma) \mathbf{x}$ for each $\mathbf{x}$ such that $\|\mathbf{x}\|=\gamma$, and for the remaining points $\psi$ is a linear extension on each segment $\left\{t \mathbf{x} \mid t \in[0,1], \mathbf{x} \in \partial B^{n}\right\}$. Then $\psi$ is the desired homeomorphism. 
A similar argument establishes the next lemma. It enables us to obtain homeomorphisms like $\psi: B^{n} \rightarrow B^{n}$ of Lemma 12.6 as the composition of finitely many homeomorphisms, each one moving points less than a predetermined constant amount.

Lemma 12.7. Consider $B^{n}$ as a cone $O * \partial B^{n}$. Suppose $\gamma \in(0,1), \eta \in$ $(0, \gamma), Z$ and $Z^{\prime}$ are compact subsets of $\partial B^{n}$ with $Z \supset Z^{\prime}, Y$ is a compact set in $(0 * Z) \cap \gamma B^{n}$, and $U$ is a neighborhood of $(O * Z) \cap \gamma B^{n}$. Then there is a homeomorphism $\psi: B^{n} \rightarrow B^{n}$ such that

(1) $\psi\left|\left\lceil\partial B^{n} \cup(O * Z) \cup\left(B^{n}-U\right)\right\rfloor=\mathrm{id}\right|\left\lceil\partial B^{n} \cup(O * Z) \cup\left(B^{n}-U\right)\right\rfloor$,

(2) $\varrho(\psi$, id $)<\eta$,

(3) $\psi(Y) \subset(\gamma-\eta)(O * Z) \cup\left[N\left(O * Z^{\prime} ; \eta\right) \cap(O * Z)\right]$.

Proposition 12.8. Suppose $K$ is a 2-complex $L C C^{1}$ embedded in $M$, $f: M \rightarrow X$ is a cell-like mapping that is $1-1$ on $K$ and that induces a decomposition $G_{f}=\left\{f^{-1}(x) \mid x \in X\right\}$ for which the nondegeneracy set $N_{f}$ has embedding dimension at most 2 and satisfies $f\left(N_{f}\right) \subset f(K)$, and $V$ is a neighborhood of $f\left(N_{f}\right)$. Then $f$ can be approximated, arbitrarily closely, by a cell-like map $F: M \rightarrow M$ satisfying

(1) $F$ is $1-1$ on $K$,

(2) $F\left|\left\lceil M-f^{-1}(V)\right\rfloor=f\right|\left\lceil M-f^{-1}(V)\right\rfloor$,

(3) the nondegeneracy set $N_{F}$ has embedding dimension at most 2 ,

(4) $F\left(N_{F}\right) \subset F\left(K-K^{(0)}\right)$.

Proof. Let $\mathcal{G}=\left\{f^{-1} f(v) \mid v \in K^{(0)}\right\}$ together with all singletons from the rest of $M$ denote the decomposition induced by $f$ over the vertices of $f(K)$. The key step involves showing that $\mathcal{G}$ strongly $\varepsilon$-shrinks while fixing $K$. Identify the vertices $\left\{v_{1}, \ldots, v_{k}\right\}$ in $K^{(0)}$ and apply Proposition 12.5 to obtain pairwise disjoint 5 -cells $\left\{B_{1}, \ldots, B_{k}\right\}$ such that

$$
f^{-1} f\left(v_{i}\right) \subset \operatorname{int}\left(B_{i}\right) \subset B_{i} \subset f^{-1}\left(V \cap N\left(f\left(v_{i}\right) ; \varepsilon / 2\right)\right)
$$

with $B_{i} \cap K \subset N\left(v_{i} ; \varepsilon\right)$ a cone neighborhood of $v_{i}$ in $K$. Lemma 2.5 ensures that $\mathcal{G}$ strongly $\varepsilon$-shrinks fixing $K$.

As a result, Proposition 12.4 promises a map $\mu: M \rightarrow M$ satisfying

- $\mu|\lceil K\rfloor=\operatorname{id}|\lceil K\rfloor$,

- $\mu$ is supported in $f^{-1}(V)$,

- $\mathcal{G}=\left\{\mu^{-1}(x) \mid x \in M\right\}$,

- $\varrho\left(f \mu^{-1}, f\right)<\varepsilon$.

The required cell-like map is $F=f \mu^{-1}$, which obviously is 1-1 over $F\left(K_{0}\right) \cup$ $(X-F(K))$. The nondegeneracy set of $F$ is locally equivalent to that of $f$, so $\operatorname{dem}\left(N_{F}\right) \leq 2$, since embedding dimension is locally determined. 
Proposition 12.9. Given the data from the conclusion of Proposition 12.8, the map $F$ can be approximated, arbitrarily closely, by a cell-like map $\widehat{F}: M \rightarrow X$ satisfying

(1) $\widehat{F}$ is $1-1$ on $K$,

(2) $\widehat{F}\left|\left\lceil M-F^{-1}(U)\right\rfloor=F\right|\left\lceil M-F^{-1}(U)\right\rfloor$,

(3) the nondegeneracy set $N_{\widehat{F}}$ has embedding dimension at most 2,

(4) $\widehat{F}\left(N_{\widehat{F}}\right) \subset \widehat{F}\left(K-K^{(1)}\right)$.

We leave proof details to the reader. The argument is essentially the same as that for the forthcoming Proposition 12.11, except that some of the cone neighborhoods in $K$ employed in the latter are simpler to describe (being 2-cells). Besides, for the decompositions arising in the Main Application, we can avoid use of 12.9 but must use Proposition 12.11.

Definition 12.10. Given a closed subset $K$ of $M$, a usc decomposition $G$ of $M$, with decomposition map $\pi: M \rightarrow M / G$, and $\varepsilon>0$, we say that an amalgamation $G^{*}$ of $G$ strongly $\varepsilon$-shrinks fixing $K$ if, for every neighborhood $U$ of $N_{G^{*}}$, there exists a homeomorphism $h: M \rightarrow M$ such that $h$ is supported in $U, h$ fixes $K, \varrho(\pi h, \pi)<\varepsilon$ and $\operatorname{diam}\left(h\left(g^{*}\right)\right)<\varepsilon$ for each $g^{*} \in G^{*}$.

Proposition 12.11. Suppose $G$ is a usc decomposition of a compact metric space $S$ such that for each $\varepsilon>0$, there exists an $\varepsilon$-amalgamation $G^{*}$ of $G$ that strongly $\varepsilon$-shrinks fixing the compact set $K$. Then $G$ is shrinkable fixing $K$.

Proof. This follows by a straightforward adaptation of [11, Theorem 20.1].

Proposition 12.12. Suppose that $K$ is a finite 2-complex $L C C^{1}$ embedded in $M, G$ is a 0-dimensional cell-like decomposition of $M$, the decomposition map $\pi: M \rightarrow M / G$ is 1-1 on $K, N_{G} \subset \pi^{-1}\left(\pi\left(K-K^{(1)}\right)\right)$, and $\operatorname{dem}\left(N_{G}\right) \leq 2$. Then for every $\varepsilon>0$ and every neighborhood $U$ of $N_{G}, G$ has an $\varepsilon$-amalgamation $G^{*}$ so that $H_{G^{*}}$ forms a null sequence and, for each $g^{*} \in H_{G^{*}}, \operatorname{dem}\left(g^{*}\right) \leq 2, g^{*} \subset U, g^{*} \cap K=g^{*} \cap\left(K-K^{(1)}\right)$ is a cell-like set and $\operatorname{diam}\left(g^{*} \cap K\right)<\varepsilon$.

Proof. Let $C=K \cap N_{G}$; it is a 0-dimensional, $\sigma$-compact set, each point of which meets exactly one element of $G$. Express $C$ as $\bigcup_{k} C_{k}$, with each $C_{k}$ compact. Cover $C_{1}$ by the interiors of finite number of pairwise disjoint 2-cells $D_{1,1}, \ldots, D_{1, n(1)}$ in $U \cap\left(K-K^{(1)}\right)$, where each $D_{1, i}$ and each $\pi\left(D_{1, i}\right)$ has diameter less than $\varepsilon$ and $\partial D_{1, i} \cap C=\emptyset$. For $k=2,3, \ldots$ recursively determine a family $D_{k, 1}, \ldots, D_{k, n(k)}$ of pairwise disjoint 2-cells in $U \cap\left(K-K^{(1)}\right)$ which miss all the previously determined $D_{j, i}$, whose interiors cover $C_{k}-\bigcup_{j=1}^{k-1} \bigcup_{i=1}^{n(j)} D_{j, i}$, whose boundaries miss $C$, and which satisfy 
$\operatorname{diam}\left(D_{k, i}\right)<\varepsilon / 2^{k}$ and $\operatorname{diam}\left(\pi\left(D_{k, i}\right)\right)<\varepsilon / 2^{k}$. Define $G^{*}$ as the decomposition of $M$ whose nondegenerate elements are the various sets $\pi^{-1} \pi\left(D_{k, i}\right)$.

Proposition 12.13. Suppose that $K$ is a finite 2-complex $L C C^{1}$ embedded in $M, G$ is a 0-dimensional cell-like decomposition of $M$, the decomposition map $\pi: M \rightarrow M / G$ is 1-1 on $K, N_{G} \subset \pi^{-1}(\pi(K))$, and $\operatorname{dem}\left(N_{G}\right) \leq 2$. Then $G$ is strongly shrinkable fixing $K$.

Proof. From Proposition 12.9 we may assume that (after an approximation to the given cell-like map) $N_{G}$ misses $K^{(1)}$.

Fix $\varepsilon>0$ and a neighborhood $U$ of $N_{G}$. Apply Proposition 12.12 to obtain an $\varepsilon / 3$-amalgamation $G^{*}$ of $G$ whose nondegenerate elements form a null sequence such that $\operatorname{diam}\left(g^{*} \cap K\right)<\varepsilon / 3, g^{*} \subset U$, and $\operatorname{dem}\left(g^{*}\right) \leq 2$ for each $g^{*} \in G^{*}$. By Proposition 12.11 it suffices to show that $G^{*}$ strongly $\varepsilon$-shrinks fixing $K$.

Let $\Lambda=\left\{g_{1}, \ldots, g_{k}\right\}$ include all elements of $G^{*}$ having diameter at least $\varepsilon / 3$. Specify a set $\left\{U_{1}, \ldots, U_{k}\right\}$ of pairwise disjoint $G^{*}$-saturated open sets such that $\operatorname{diam}\left(\pi\left(U_{j}\right)\right)<\varepsilon, g_{j} \subset U_{j} \subset U$, and any other $g^{*} \in G^{*}-\Lambda$ satisfies $\operatorname{diam}\left(g^{*}\right)<\varepsilon / 3$. Use Proposition 12.5 to obtain a collection $\left\{B_{1}, \ldots, B_{k}\right\}$ of disjoint 5-cells such that $g_{j} \subset \operatorname{int}\left(B_{j}\right) \subset B_{j} \subset U_{j}$ and $B_{j} \cap K$ is a 2-cell $D_{j} \subset K$ with $\operatorname{diam}\left(D_{j}\right)<\varepsilon / 3$. Choose a point $O_{j} \in \operatorname{int}\left(D_{j}\right)-N_{G^{*}}$. We follow a shrinking procedure very similar to that found in [11, Proposition 22.1], except in this case the shrinking also fixes $K$.

The following lemma is needed:

Lemma 12.14. Consider $B^{n}$ as a cone $O * \partial B^{n}$. Suppose that $Y$ is a compact set in $\operatorname{int}\left(B^{n}\right)$ missing $O$ and $Z$ is a compact set in $\partial B^{n}$ such that $\operatorname{dem}(Z) \leq \operatorname{dem}(Y)-1$. Then there is a homeomorphism $\varphi: B^{n} \rightarrow B^{n}$ fixing $O * Z$ so that if $O * X$ is the union of all cone segments meeting $\varphi(Y)$, then $\operatorname{dem}(O * X) \leq \operatorname{dem}(Y)+1$.

Proof. Let $\operatorname{dem}(Y)=k$ so that $\operatorname{dem}(Z) \leq k-1$. Choose a sequence $\left\{T_{i}\right\}$ of triangulations of $\partial B^{n}$ with mesh going to zero so that the $(n-k-2)$ skeleton of $T_{i}$ misses $Z$. Let $P_{i}$ denote the geometric cone in $B^{n}$ from $O$ over the $(n-k-2)$ skeleton of $T_{i}$. Then applying the hypothesis on embedding dimension, we can adjust $Y$ to miss $\bigcup P_{i}$ by a homeomorphism $\varphi$ which fixes $O * Z$. Let $X$ be the projection of $\varphi(Y)$ from $O$ to $\partial B^{n}$. Then

$$
\operatorname{dim} X \leq(n-1)-(n-k-2)-1=k
$$

moreover, $\varphi(Y) \subset O * X$ and $\operatorname{dem}(O * X) \leq k+1=\operatorname{dem}(Y)+1$.

Regard $B_{j}$ as $O_{j} * \partial B_{j}$ and $Z_{j}^{\prime} \subset Z_{j} \subset \partial B_{j}$ with

$$
g_{j} \subset O_{j} * Z_{j}, \quad \operatorname{dem}\left(Z_{j}\right)=2, \quad K \cap B_{j}=D_{j}=O_{j} * Z_{j}^{\prime} .
$$

Set $A_{j}=O_{j} * Z_{j}$. Choose $\eta>0$ so that $\eta$-diameter subsets of $O_{j} * \partial B_{j}$ have 
image diameter less than $\varepsilon / 3$ in $B_{j}$, and assume $\eta$ to be so small that the $\eta$-neighborhood of $O * Z^{\prime}$ corresponds to a set in $B_{j}$ of diameter less than $\varepsilon$ near $D_{j}$. Decreasing $\eta$ if necessary, choose an integer $m>0$ such that $\gamma=(m+1) \eta<1$ and $g_{j}$ corresponds to a subset of $\gamma A_{j}$. Set $W_{0}=U$. Apply Lemma 12.7 in each $B_{j}$ and extend over $M-\bigcup_{j} B_{j}$ via the identity to obtain a homeomorphism $\Psi_{1}: M \rightarrow M$ satisfying

(1) $\Psi_{1}\left|\left\lceil K \cup\left(M-W_{0}\right)\right\rfloor=\mathrm{id}\right|\left\lceil K \cup\left(M-W_{0}\right)\right\rfloor$,

(2) $\varrho\left(\Psi_{1}\right.$, id $)<\varepsilon / 3$

(3) $\Psi_{1}\left(g_{j}\right) \subset\left[(\gamma-\varepsilon / 3) A_{j} \cup N\left(O_{j} * Z_{j}^{\prime} ; \varepsilon / 3\right)\right] \cap\left(O_{j} * Z_{j}\right)$.

Now if the images under $\Psi_{1}$ of all $g^{*} \in G^{*}-\Lambda$ that meet $\bigcup_{j}(\gamma-\varepsilon / 3) A_{j}$ would have diameter less than $\varepsilon / 3$, we could choose a neighborhood $W_{1} \subset$ $W_{0}$ of $\bigcup_{j}(\gamma-\eta) A_{j}$ such that if $g^{*} \in G^{*}-\Lambda$ and $\Psi_{1}\left(g^{*}\right) \cap W_{1} \neq \emptyset$, then $\operatorname{diam}\left(\Psi_{1}\left(g^{*}\right)\right)<\eta$, and we could apply Lemma 12.7 again to obtain a second homeomorphism $\Psi_{2}: M \rightarrow M$ so $\Psi_{2} \Psi_{1}$ shrinks $\bigcup_{j} \gamma A_{j}$ to $(\gamma-2 \varepsilon / 3) A_{j}$. However, that is too much to expect, in general, and to address that obstacle we use the following:

Lemma 12.15. Suppose $K$ is a finite 2-complex $L C C^{1}$ embedded in $M$, $\varepsilon>0$, and $G^{*}$ is a cell-like usc decomposition of $M$ for which $H_{G^{*}}$ forms a null sequence of compacta, where $\operatorname{dem}\left(g^{*}\right) \leq 2$ and $g^{*} \cap K=g^{*} \cap\left(K-K^{(1)}\right)$ is a cell-like set of diameter less than $\varepsilon$ for each $g^{*} \in G^{*}, A$ is a compact 3-dimensional polyhedron in $M$, and $g_{t} \in G^{*}$ satisfies $\operatorname{diam}\left(g_{t} \cap K\right)<\varepsilon / 3$. Then there exist a neighborhood $W$ of $g_{t}$ and a homeomorphism $\psi: M \rightarrow M$ such that

$$
\begin{aligned}
& \text { - } \psi|\lceil K \cup(M-W)\rfloor=\operatorname{id}|\lceil K \cup(M-W)\rfloor, \\
& \text { - } \operatorname{diam}\left(\psi\left(g^{*}\right)\right)<\varepsilon / 3 \text { for all } g^{*} \in G^{*} \text { with } \psi\left(g^{*}\right) \cap W \cap A \neq \emptyset \text {. }
\end{aligned}
$$

Lemma 12.15 is proved in much the same fashion as [11, Lemma 22.3], with additional stipulations about not moving points of $K$. (Unlike in [11], there is no need here for an inductive argument, because embedding dimension considerations make the result quite obvious when $\operatorname{dem}(A)<3$.) Details are left to the reader.

Returning to the proof of Proposition 12.13, we consider the finite collection of elements $g^{*} \in G^{*}-\Lambda$ whose images under $\Psi_{1}$ have diameter at least $\varepsilon / 3$ (and, hence, diameter at most $\varepsilon$ ). Apply Lemma 12.15 , with $\Psi_{1}\left(G^{*}\right)$ and positive number $\varepsilon / 3$, in very small neighborhoods (i.e., in $\varepsilon$-diameter neighborhoods) of such $\Psi_{1}\left(g^{*}\right)$ to obtain a resulting homeomorphism $\psi_{1}: M \rightarrow M$ such that

(4) $\psi_{1}\left|\left\lceil K \cup\left(M-W_{0}\right)\right\rfloor=\mathrm{id}\right|\left\lceil K \cup\left(M-W_{0}\right)\right\rfloor$,

(5) $\psi_{1}\left|\left\lceil\Psi_{1}\left(g^{*}\right)\right\rfloor=\mathrm{id}\right|\left\lceil\Psi_{1}\left(g^{*}\right)\right\rfloor$ for all $g^{*} \in \Lambda$,

(6) $\operatorname{diam}\left(\psi_{1} \Psi_{1}\left(g^{*}\right)\right)<\varepsilon / 3$ for all $g^{*} \in G^{*}-\Lambda$ with $\psi_{1} \Psi_{1}\left(g^{*}\right) \cap \bigcup_{j} A_{j} \neq \emptyset$. 
Now choose a neighborhood $W_{1} \subset W_{0}$ of $\bigcup_{j}(\gamma-\varepsilon / 3) A_{j}$ such that if $g^{*} \in G^{*}-\Lambda$ and $\psi_{1} \Psi_{1}\left(g^{*}\right) \cap W_{1} \neq \emptyset$, then $\operatorname{diam}\left(\psi_{1} \Psi_{1}\left(g^{*}\right)\right)<\varepsilon / 3$. Again apply Lemma 12.7 to obtain a second homeomorphism $\Psi_{2}: M \rightarrow M$ so that

(7) $\Psi_{2} \mid\left\lceil K \cup\left(M-W_{1}\right)\right\rfloor=$ id $\mid\left\lceil K \cup\left(M-W_{1}\right)\right\rfloor$,

(8) $\varrho\left(\Psi_{2}\right.$,id $)<\varepsilon / 3$

(9) $\Psi_{2}\left((\gamma-\varepsilon / 3) A_{j}\right) \subset(\gamma-2 \varepsilon / 3) A_{j} \cup\left[N\left(O_{j} * Z_{j}^{\prime} ; \varepsilon / 3\right) \cap A_{j}\right], j=1,2, \ldots$

At this point consider the finite collection of elements $g^{*} \in G^{*}-\Lambda$ whose images under $\Psi_{2} \psi_{1} \Psi_{1}$ have diameter between $\varepsilon / 3$ and $\varepsilon$. Apply Lemma 12.15 , with $\Psi_{2} \psi_{1} \Psi_{1}\left(G^{*}\right)$ and positive number $\varepsilon / 3$, in very small neighborhoods of such $\Psi_{2} \psi_{1} \Psi_{1}\left(g^{*}\right)$ to obtain a resulting homeomorphism $\psi_{2}: M \rightarrow M$ such that

(10) $\psi_{2}\left|\left\lceil K \cup\left(M-W_{1}\right)\right\rfloor=\mathrm{id}\right|\left\lceil K \cup\left(M-W_{1}\right)\right\rfloor$,

(11) $\psi_{2} \mid\left\lceil\Psi_{2} \psi_{1} \Psi_{1}\left(g^{*}\right)\right\rfloor=$ id $\mid\left\lceil\Psi_{2} \psi_{1} \Psi_{1}\left(g^{*}\right)\right\rfloor$ for all $g^{*} \in \Lambda$,

(12) $\operatorname{diam}\left(\psi_{2} \Psi_{2} \psi_{1} \Psi_{1}\left(g^{*}\right)\right)<\varepsilon / 3$ for all $g^{*} \in G^{*}-\Lambda$ with $\psi_{2} \Psi_{2} \psi_{1} \Psi_{1}\left(g^{*}\right) \cap$ $W_{1} \cap \bigcup_{j} A_{j} \neq \emptyset$.

Repeat this procedure, producing successive homeomorphisms $\Psi_{1}, \psi_{1}$, $\Psi_{2}, \psi_{2}, \ldots, \psi_{m-1}, \Psi_{m}$. The composition $F=\Psi_{m} \psi_{m-1} \Psi_{m-1} \cdots \psi_{2} \Psi_{2} \psi_{1} \Psi_{1}$ has the desired effect. Not only does it shrink each $g_{j} \in \Lambda \subset G^{*}$ to small size while keeping $K$ fixed, it does the shrinking with control: if

$$
\Psi_{s} \psi_{s-1} \Psi_{s-1} \cdots \psi_{1} \Psi_{1}\left(g^{*}\right), \quad \text { where } g^{*} \in G^{*}-\Lambda,
$$

becomes dangerously large (that is, of diameter between $\varepsilon / 3$ and $\varepsilon$ ), either $\psi_{s}$ rectifies that problem or $\psi_{s}$ pushes that image off $\bigcup_{j} A_{j}$ and allows later compressions of these $g_{j} \in \Lambda$ across $A_{j}$ to sweep past without further affecting any moderately large images. In other words, the large elements of $G^{*}$ are shrunk to small size, without allowing any of the originally small elements to become too big.

Proof of Theorem 12.1. This argument is quite similar to that of [11, Theorem 23.2], with minor notational changes. It is so fundamental that we present some of the details.

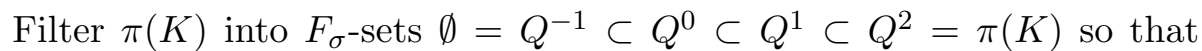
$\operatorname{dim}\left(Q^{i}\right) \leq i$ and $\operatorname{dim}\left(Q^{i}-Q^{i-1}\right)=0$ [11, Lemma 23.1]. We shall apply previous results from this section to successively approximate $\pi$ by celllike maps that are 1-1 over $Q^{0}, Q^{1}, Q^{2}$, respectively. The Claim is that for $i=0,1,2$ and $\varepsilon>0$ there exists a cell-like map $F_{i}: M \rightarrow M / G$ such that $\varrho\left(F_{i}, \pi\right)<(i+1) \varepsilon, F_{i}$ is $1-1$ over $Q^{i} \cup(M-\pi(K)), F_{i} \mid\lceil K \cup(M-U)\rfloor$ $=\pi \mid\lceil K \cup(M-U)\rfloor$, and the nondegeneracy set of $F_{i}$ has embedding dimension at most 2. Once the claim is established, the final $F_{2}$ will be a homeomorphism that approximates $\pi$ and agrees with $\pi$ on $K \cup(M-U)$, as required. 
To begin, express $Q^{0}$ as the countable union of compact 0-dimensional sets $Z_{j}$. Choose open subsets $V_{1}, V_{2}, \ldots$ of $M / G$ with $V_{i+1} \subset V_{i}, \pi^{-1}\left(V_{1}\right) \subset$ $U$ and $\pi(K) \supset \bigcap_{j} V_{j} \supset Q^{0}$. We shall produce cell-like maps $f_{j}: M \rightarrow$ $M / G, j \in\{0,1,2, \ldots\}$, with $f_{0}=\pi$, satisfying the following conditions:

(1) $\varrho\left(f_{j+1}, f_{j}\right)<\varepsilon / 2^{j+1}$;

(2) $f_{j}$ is $1-1$ over $(M / G-\pi(K)) \cup \bigcup_{k=1}^{j} Z_{k}$;

(3) there exists a PL triangulation $T_{j}$ of $M$ of mesh less than $2^{-j}$ whose 2-skeleton $T^{(2)}$ misses the nondegeneracy set of $f_{j}$;

(4) $f_{j+k}\left|\left\lceil T_{j}^{(2)} \cup f_{j}^{-1}\left(Z_{j}\right) \cup\left(M-f_{j}^{-1}\left(V_{j}\right)\right)\right\rfloor=f_{j}\right|\left\lceil T_{j}^{(2)} \cup f_{j}^{-1}\left(Z_{j}\right) \cup\right.$ $\left.\left(M-f_{j}^{-1}\left(V_{j}\right)\right)\right\rfloor$ for $j, k \in\{1,2, \ldots\}$;

(5) for $x \in M-\left(T_{j}^{(2)} \cup f_{j}^{-1}\left(Z_{j}\right)\right)$ and $j, k \in\{0,1,2, \ldots\}$,

$$
\varrho\left(f_{j+k+1}(x), f_{j+k}(x)\right) \leq 4^{-k} \varrho\left(f_{j+k}(x), f_{j}\left(T_{j}^{(2)}\right) \cup Z_{j}\right) .
$$

Before describing their construction, we describe their purpose. Condition (1) ensures that $\left\{f_{j}\right\}$ is a Cauchy sequence of maps converging to a map $F_{0}$ close to $\pi$, where $F_{0}$ is necessarily cell-like, by [11, Theorem 17.4]. Condition (2) requires $f_{j}$ to be $1-1$ over $Z_{j}$, condition (4) maintains the same action over $Z_{j}$ by subsequent maps, and condition (5) provides critical controls ensuring the same feature holds in the limiting map $F_{0}$, by preventing points of $M-f_{j}^{-1}\left(Z_{j}\right)$ from being sent to $Z_{j}$ by $F_{0}$. Consequently, $F_{0}$ will be $1-1$ over $Q_{0}$. Similarly, $f_{j}$ is $1-1$ over $M / G-f_{j}^{-1}\left(V_{j}\right)$, subsequent maps $f_{j+k}$ agree with $f_{j}$ over $M-f_{j}^{1}\left(V_{j}\right)$ by $(4)$, and thus $F_{0}$ is $1-1$ over $M / G-\bigcap V_{j} \subset M / G-\pi(K)$. Very important among all these properties, condition (3) identifies a fine-meshed triangulation whose 2-skeleton $T_{j}^{(2)}$ misses the nondegeneracy set of $f_{j}$, condition (4) maintains the same action relative to $T_{j}^{(2)}$ by subsequent maps, and condition (5) prevents any other points of $M$ from being sent to $F_{0}\left(T_{j}^{(2)}\right)=f_{j}\left(T_{j}^{(2)}\right)$ under $F_{0}$. As a result, the nondegeneracy set of $F_{0}$ will be contained in $M-\bigcup_{j} T_{j}^{(2)}$, causing it to have embedding dimension at most 2. Consequently, construction of the $\left\{f_{j}\right\}$ will establish the claim for the $i=0$ case.

To perform the construction of the first new map, $f_{1}$, observe that the decomposition $G(1)$ induced over $Z_{1}$ by $f_{0}$, namely,

$$
G(1)=\left\{f_{0}^{-1}(z) \mid z \in Z_{1}\right\} \text { plus all singletons from } M-f_{0}^{-1}\left(Z_{1}\right),
$$

is a 0 -dimensional, cell-like decomposition of $M$ satisfying the hypothesis of Proposition 12.13. Applying Propositions 12.13 and 12.4 we obtain a map $\mu_{1}: M \rightarrow M$ such that $\mu_{1}$ realizes $G(1)$ (in other words, that $G(1)=$ $\left.\left\{\mu_{1}^{-1}(x) \mid x \in M\right\}\right), \mu_{1}$ moves no point of $T_{1}^{(2)}$ or outside $U_{1}$, and $\mu_{1}$ is limited by the inverse image under $f_{0}=h$ of an open cover consisting of sets of diameter less than $\varepsilon / 2$. Define $f_{1}=f_{0} \mu_{1}^{-1}$. Obviously $f_{1}$ is a well-defined map; 
most notably, it is 1-1 not only over $Z_{1}$ but also over all points $x \in M / G$ for which $f_{0}^{-1}(x)$ is a singleton. Its definition ensures that any nontrivial point preimage under $f_{1}$ is homeomorphic to the preimage of the same point under $f_{0}$, so $f_{1}$ is cell-like. One can produce the required p.l. triangulations $T_{1}$ of $M$ to ensure that $f_{1}$ satisfies conditions (1) through (3). Construction of subsequent maps $f_{2}, f_{3}, \ldots$ proceeds in similar fashion, controlled to ensure satisfaction of conditions (1) through (5). See [11, pp. 173-174] for further details.

Now, on to the large-scale iteration. Assume $F_{i}: M \rightarrow M / G(i=0,1)$ is a cell-like map satisfying the statements of the Claim. The new goal is to produce another cell-like map $F_{i+1}: M \rightarrow M / G, \varepsilon$-close to $F_{i}$ and 1-1 over $(M / G-\pi(K)) \cup Q^{i+1}$. Toward that end, express $Q^{i+1}$ as a countable union of compact sets $Z_{1}, Z_{2}, \ldots$ Determine open subsets $V_{1} \supset V_{2} \supset \ldots$ such that $\pi(K) \supset \bigcap_{j} V_{j} \supset Q^{i+1}$ and $\pi^{-1}\left(V_{1}\right) \subset U$. Exactly as in the $i=0$ case, we find cell-like maps $f_{j}: M \rightarrow M / G, j \in\{0,1,2, \ldots\}$, with $f_{0}=F_{i}$, satisfying conditions (1) through (5) as before, except for condition (2) which is upgraded to:

$\left(2^{\prime}\right) f_{j}$ is $1-1$ over $(M / G-\pi(K)) \cup Q^{i} \cup \bigcup_{k=1}^{j} Z_{k}$.

The key for starting and for iterating is that the decomposition induced by $f_{0}=F_{i}$ over $Z_{1}$ is 0 -dimensional, since its nondegeneracy set is a subset of $Z_{1}-Q^{i} \subset Q^{i+1}-Q^{i}$. This exposes the principal benefit of the filtration. Use Propositions 12.13 and 12.4, as before, to approximate $f_{0}$ by a new cell-like map $f_{1}$ which is $1-1$ over $(M / G-\pi(K)) \cup Q^{i} \cup Z_{1}$. Continue the iteration, obtaining maps $f_{2}, f_{3}, \ldots$ converging to the desired $F_{i+1}$, and completing the verification of the iterative step. This finishes the proof of the Claim and of Theorem 12.1.

Corollary 12.16. Let $K$ be a 2-complex $L C C^{1}$ embedded in $M$. Suppose $h: M \rightarrow M$ is a cell-like map that is $1-1$ on $K, G_{h}$ is the induced decomposition, $h\left(N_{h}\right) \subset h(K)$, and $\operatorname{dem}\left(N_{h}\right) \leq 2$. Then for each neighborhood $V$ of $h(K)$ and each $\varepsilon>0$ there exists a homeomorphism $\Phi: M \rightarrow M$ such that

(1) $\Phi|\lceil K\rfloor=h|\lceil K\rfloor$,

(2) $\Phi=h$ outside $h^{-1}(V)$,

(3) $\varrho(\Phi, h)<\varepsilon$.

13. Proof of the main theorem. We are now ready to prove the main theorem. In particular, we prove a restatement of Theorem 6.1 in the language of decompositions.

Theorem 13.1 (Main Theorem). Let $G$ be a cell-like usc decomposition of a compact, p.l. 5-manifold $M$ for which $\operatorname{dim}(M / G)<\infty$ and the associated decomposition map $\pi: M \rightarrow M / G$ has the disjoint disks property. 
Then $\pi$ can be approximated by a cell-like map $\Psi: M \rightarrow M / G$ such that $\operatorname{dem}\left(N_{\Psi}\right) \leq 2$.

Proof. By Theorem 7.3, $\pi: M \rightarrow M / G$ can be approximated by an $\mathrm{LCC}^{1}$ refined map. Without loss of generality, suppose that $\pi$ is $\mathrm{LCC}^{1}$ refined over $\mathcal{A}$. Fix $\varepsilon>0$ and set $\lambda_{0}=\pi$. Let $\left\{K_{i}\right\}$ be a sequence of pairwise disjoint, $\mathrm{LCC}^{1}$ embedded, finite 2-complexes that are the 2-skeleta of triangulations of $M$ whose mesh tends to zero. By Corollary 11.4 there is a cell-like map $h_{1}: M \rightarrow M$, the end of a pseudo-isotopy moving $K_{1}$ off $N_{G}$, so that $\varrho\left(\lambda_{0} h_{1}, \lambda_{0}\right)<\varepsilon / 2, h_{1}$ is $1-1$ on $K_{1}, h_{1}\left(N_{h_{1}}\right) \subset h_{1}\left(K_{1}\right)$, and $\operatorname{dem}\left(N_{h_{1}}\right) \leq 2$. Corollary 12.16 then provides a homeomorphism $\Phi_{1}: M \rightarrow M$ such that $\Phi_{1}\left|\left\lceil K_{1}\right\rfloor=h_{1}\right|\left\lceil K_{1}\right\rfloor$ and $\varrho\left(\lambda_{0} \Phi_{1}, \lambda_{0}\right)<\varepsilon / 2$. Now $\lambda_{1}=\lambda_{0} \Phi_{1}$ is $1-1$ over $\lambda_{1}\left(K_{1}\right)$, since $\Phi_{1}\left(K_{1}\right)=h_{1}\left(K_{1}\right)$ misses $N_{G}$. To continue one must observe that $\lambda_{1}: M \rightarrow M / G$ is $\mathrm{LCC}^{1}$ refined over $\mathcal{A}$. The rest of the proof proceeds like that of [11, Theorem 24.3]. In the next step, for instance, we apply Corollary 11.4 again to locate a cell-like map $h_{2}: M \rightarrow M$, the end of a pseudo-isotopy supported in $M-K_{1}$ and moving $K_{2}$ off the nondegeneracy set $N_{\lambda_{1}}$ of $\lambda_{1}$, so that $\varrho\left(\lambda_{1} h_{2}, \lambda_{1}\right)<\varepsilon / 4, h_{2}$ is 1-1 on $K_{2}, h_{2}\left(N_{h_{2}}\right) \subset h_{2}\left(K_{2}\right)$, and $\operatorname{dem}\left(N_{h_{2}}\right) \leq 2$. Corollary 12.16 then yields a homeomorphism $\Phi_{2}: M \rightarrow$ $M$ supported in $M-K_{1}$ such that $\Phi_{2}\left|\left\lceil K_{2}\right\rfloor=h_{2}\right|\left\lceil K_{2}\right\rfloor$ and $\varrho\left(\lambda_{1} \Phi_{2}, \lambda_{1}\right)<$ $\varepsilon / 4$. Here $\lambda_{2}=\lambda_{1} \Psi_{2}$ is $1-1$ over $\lambda_{2}\left(K_{2}\right)$, since $\Phi_{2}\left(K_{2}\right)=h_{2}\left(K_{2}\right)$ misses $N_{\lambda_{1}}$. In this fashion we build cell-like maps $\lambda_{i}: M \rightarrow M / G, i \in\{1,2, \ldots\}$, where $\lambda_{i}$ is 1-1 over $\lambda_{i}\left(K_{i}\right)$, and where this collection of maps forms a Cauchy sequence converging to a cell-like map $\Psi: M \rightarrow M / G$ with $\varrho\left(\Psi, \pi=\lambda_{0}\right)<\varepsilon$. Besides maintaining controls to ensure $\left\{\lambda_{i}\right\}$ is a Cauchy sequence, motion is carefully regulated to ensure that $\lambda_{i+k}\left|\left\lceil K_{i}\right\rfloor=\lambda_{i}\right|\left\lceil K_{i}\right\rfloor$ and $\lambda_{i+k}$ is $1-1$ over $\lambda_{i+k}\left(K_{i}\right)$. With even more careful control the same feature is preserved in the limit, i.e., $\Psi$ is $1-1$ over $\Psi\left(K_{i}\right)$ and hence $1-1$ over $\Psi\left(\bigcup_{i=1}^{\infty} K_{i}\right)$. It follows that $\operatorname{dem}\left(N_{\Psi}\right) \leq 2$.

The cell-like approximation in dimension 5 now follows as a corollary, as explained in Sections 4 and 5.

Theorem 13.2 (Cell-like approximation theorem in dimension 5). Suppose $G$ is a cell-like decomposition of an 5 -manifold $M$. Then $G$ is shrinkable if and only if $M / G$ is finite-dimensional and has the disjoint disks property.

\section{References}

[1] E. Akin, Manifold phenomena in the theory of polyhedra, Trans. Amer. Math. Soc. 143 (1969), 413-473.

[2] R. H. Bing, Radial engulfing, in: Conference on the Topology of Manifolds (East Lansing, MC, 1967), Prindle, Weber \& Schmidt, Boston, MA, 1968, 1-18. 
[3] J. L. Bryant, Piecewise linear topology, in: Handbook of Geometric Topology, Elsevier, 2002, 219-259.

[4] J. L. Bryant, S. Ferry, W. Mio, and S. Weinberger, Topology of homology manifolds, Ann. of Math. (2) 143 (1996), 435-467.

[5] J. L. Bryant and C. L. Seebeck, Locally nice embeddings in codimension three, Quart. J. Math. Oxford (2) 21 (1970), 265-272.

[6] J. W. Cannon, The recognition problem: what is a topological manifold?, Bull. Amer. Math. Soc. 84 (1978), 832-866.

[7] -, Shrinking cell-like decomposition of manifolds. Codimension three, Ann. of Math. (2) 110 (1979), 83-112.

[8] J. I. Cobb, Taming almost PL embeddings in codimension 3, Abstract 68T-241, Notices Amer. Math. Soc. (2) 15 (1968), 371.

[9] M. M. Cohen, Simplicial structures and transverse cellularity, Ann. of Math. (2) 85 (1967), 218-245.

[10] R. Connelly, Unknotting close polyhedra in codimension three, in: Topology of Manifolds, J. C. Cantrell (ed.), Markham, Chicago, 1970, 384-388.

[11] R. J. Daverman, Decompositions of Manifolds, AMS Chelsea, Providence, RI, 2007.

[12] R. J. Daverman and D. Repovš, General position properties that characterize 3manifolds, Canad. J. Math. 44 (1992), 234-251.

[13] R. J. Daverman and T. L. Thickstun, The 3-manifold recognition problem, Trans. Amer. Math. Soc. 358 (2006), 5257-5270.

[14] A. N. Dranishnikov, On a problem of P. S. Aleksandrov, Mat. Sb. (N.S.) 135 (177) (1988), 551-557, 560 (in Russian); English transl.: Math. USSR-Sb. 63 (1989), 539545.

[15] J. Dydak and J. J. Walsh, Infinite-dimensional compacta having cohomological dimension two: an application of the Sullivan conjecture, Topology 32 (1993), 93-104.

[16] R. D. Edwards, The topology of manifolds and cell-like maps, in: Proc. Internat. Congress Math. (Helsinki, 1978), Acad. Sci. Fennica, Helsinki, 1980, 111-127.

[17] J. Hollingsworth and R. B. Sher, Triangulating neighborhoods in topological manifolds, General Topology Appl. 1 (1971), 345-348.

[18] J. F. P. Hudson, Extending piecewise-linear isotopies, Proc. London Math. Soc. (3) 16 (1966), 651-668.

[19] —, Piecewise Linear Topology, Benjamin, New York, 1969.

[20] R. T. Miller, Close isotopies on piecewise-linear manifolds, Trans. Amer. Math. Soc. 151 (1970), 597-628.

[21] F. Quinn, An obstruction to the resolution of homology manifolds, Michigan Math. J. 34 (1987), 285-291.

[22] C. P. Rourke and B. J. Sanderson, Introduction to Piecewise-Linear Topology, Ergeb. Math. Grenzgeb. 69, Springer, Berlin, 1972.

[23] F. C. Tinsley, Doctoral Dissertation, Univ. of Wisconsin at Madison, 1977.

[24] P. Wright, Radial engulfing in codimension three, Duke Math. J. 38 (1971), 295-298.

Department of Mathematics

University of Tennessee

Knoxville, TN 37996-1300, U.S.A.

E-mail: daverman@math.utk.edu
Department of Mathematics Brigham Young University Provo, UT 84602, U.S.A. E-mail: deniseh@math.byu.edu

Received 9 November 2005; in revised form 20 October 2007 